\title{
Improved cloud-phase determination of low-level liquid and mixed-phase clouds by enhanced polarimetric lidar
}

\author{
Robert A. Stillwell ${ }^{1,2}$, Ryan R. Neely III ${ }^{3,4}$, Jeffrey P. Thayer ${ }^{1}$, Matthew D. Shupe ${ }^{5,6}$, and David D. Turner \\ ${ }^{1}$ Aerospace Engineering Sciences, University of Colorado at Boulder, ECNT 320, 431 UCB, \\ University of Colorado, Boulder, CO 80309, USA \\ ${ }^{2}$ Advanced Study Program, National Center for Atmospheric Research, \\ 3450 Mitchell Lane, Bldg 1, Boulder, CO 80301, USA \\ ${ }^{3}$ School of Earth and Environment, University of Leeds, LS2 9JT, Leeds, UK \\ ${ }^{4}$ National Centre for Atmospheric Science, University of Leeds, LS2 9JT, Leeds, UK \\ ${ }^{5}$ Cooperative Institute for Research in Environmental Sciences, University of Colorado at Boulder, \\ 216 UCB, University of Colorado, Boulder, CO 80309, USA \\ ${ }^{6}$ Physical Sciences Division, Earth System Research Laboratory, National Oceanic and Atmospheric Administration, \\ 325 Broadway, Boulder, CO 80309, USA \\ ${ }^{7}$ Global Systems Division, Earth System Research Laboratory, National Oceanic and Atmospheric Administration, \\ 325 Broadway, Boulder, CO 80309, USA
}

Correspondence: Robert A. Stillwell (robert.stillwell@colorado.edu)

Received: 8 September 2017 - Discussion started: 19 September 2017

Revised: 22 December 2017 - Accepted: 3 January 2018 - Published: 14 February 2018

\begin{abstract}
The unambiguous retrieval of cloud phase from polarimetric lidar observations is dependent on the assumption that only cloud scattering processes affect polarization measurements. A systematic bias of the traditional lidar depolarization ratio can occur due to a lidar system's inability to accurately measure the entire backscattered signal dynamic range, and these biases are not always identifiable in traditional polarimetric lidar systems. This results in a misidentification of liquid water in clouds as ice, which has broad implications on evaluating surface energy budgets. The Clouds Aerosol Polarization and Backscatter Lidar at Summit, Greenland employs multiple planes of linear polarization, and photon counting and analog detection schemes, to self evaluate, correct, and optimize signal combinations to improve cloud classification. Using novel measurements of diattenuation that are sensitive to both horizontally oriented ice crystals and counting system nonlinear effects, unambiguous measurements are possible by over constraining polarization measurements. This overdetermined capability for cloud-phase determination allows for system errors to be identified and quantified in terms of their impact on cloud properties. It is shown that lidar system dynamic range ef-
\end{abstract}

fects can cause errors in cloud-phase fractional occurrence estimates on the order of $30 \%$ causing errors in attribution of cloud radiative effects on the order of 10-30\%. This paper presents a method to identify and remove lidar system effects from atmospheric polarization measurements and uses colocated sensors at Summit to evaluate this method. Enhanced measurements are achieved in this work with non-orthogonal polarization retrievals as well as analog and photon counting detection facilitating a more complete attribution of radiative effects linked to cloud properties.

\section{Introduction}

Changing Arctic conditions lead to many changes in regional surface energy and mass budgets, which have a profound impact on humans outside the region (Curry et al., 1996; Hansen et al., 2011). Locked within the Greenland Ice Sheet (GrIS) is the potential for sea level rise on the order of $7 \mathrm{~m}$ (Gregory et al., 2004), of which approximately $25 \mathrm{~mm}$ has already been contributed from 1900 to present with an increased rate of mass loss in recent years (Kjeldsen et al., 
2015). Several studies have linked variability of the surface energy and mass budgets to cloud properties and in particular low-level, liquid-only and mixed-phase ${ }^{1}$ clouds (Bennartz et al., 2013; Sherwood et al., 2014; Miller et al., 2015; Tan et al., 2016; Miller et al., 2017). The climate is sensitive to Arctic cloud macro- and microphysical properties, yet substantial gaps are present in understanding of fundamental cloud processes due to a limited set of cloud observations to which model results may be compared (Curry et al., 1996; Cesana et al., 2012; Morrison et al., 2012; Bennartz et al., 2013; Van Tricht et al., 2016).

Understanding the nature of liquid-only and mixed-phase clouds is important for understanding the surface energy budget. Mixed-phase clouds show remarkable persistence in the Arctic even though the liquid phase is colloidally unstable, possibly persisting for days to weeks given the correct synoptic conditions (Shupe et al., 2006). Furthermore, though liquid-only and mixed-phase clouds can be found up to heights of approximately $6 \mathrm{~km}$ above mean sea level (a.m.s.l.) in the Arctic, they have been found by many to be predominately low-lying with high optical thickness ${ }^{2}$ (Curry et al., 1996; Intrieri et al., 2002; Turner, 2005; Shupe et al., 2006; de Boer et al., 2009; Shupe, 2011; Shupe et al., 2013). Such characteristics make these clouds particularly hard to measure accurately from both the ground and space. Shupe et al. (2006) further note that mixed-phase clouds are an understudied component of global cloudiness resulting in their poor representation in models at all scales, a finding supported by others including Cesana et al. (2012), Pithan et al. (2014), and Kay et al. (2016). The focus of this work is the interpretation of ground-based polarimetric lidar measurements of Arctic liquid-only and mixed-phase clouds and assessing systematic measurement biases that inhibit their proper identification. While the scope of this work is confined to the Arctic, this work is informative to measurements of similar cloud types, for example present in the Antarctic.

Polarimetric lidar systems are widely deployed to the polar regions to measure cloud properties. Nott and Duck (2011) and references therein summarize more than a dozen lidar deployment sites in the Arctic and Antarctic. Polarimetric lidar data are particularly useful for cloud and aerosol studies to determine properties such as cloud phase, cloud base height, and particle orientation, as well as for broad aerosol classifications (Schotland et al., 1971; Measures, 1984; Sassen,

\footnotetext{
${ }^{1}$ This work uses the definition of mixed-phase clouds presented by Shupe et al. (2008), where a mixed-phase cloud is defined as a cloud system containing both liquid and ice water that interact via microphysical processes. The complete system must contain both liquid and ice water, but no requirement is made on the exact location or quantity of either phase.

${ }^{2}$ In this paper, "high" is taken relative to ice-only clouds existing in the same region and not to liquid clouds existing in the midlatitude or tropical regions. Here high optical thicknesses for liquid water clouds are on the order of OD 5, whereas ice is on the order of OD 1.
}

1991; Kaul et al., 2004; Fujii and Fukuchi, 2005; Weitkamp, 2005; Freudenthaler et al., 2009; Hayman and Thayer, 2012; Groß et al., 2015). The utility of lidar observations can be enhanced by using complementary measurements that grant a more complete perspective such as cloud radars, microwave radiometers, and radiosondes as done for programs like the Surface Heat Budget of the Arctic Ocean (SHEBA) (Shupe et al., 2006), the Department of Energy Atmospheric Radiation Measurement (ARM) program's atmospheric observatories (Verlinde et al., 2016), and Mixed Phase Arctic Clouds Experiment (MPACE) (Verlinde et al., 2007). Despite its utility, polarimetric lidar has limitations. Among them is the stringent requirement of linear signal operation over a large dynamic range. If not properly designed or considered, measurements can be misinterpreted casting doubt on critical measurements like cloud phase (Hayman and Thayer, 2009; Liu et al., 2009; Neely et al., 2013). For example, traditional two-channel orthogonal polarization measurements using copolarized and cross-polarized signals can not unambiguously separate systematic polarization effects and geophysical effects (Biele et al., 2000; Alvarez et al., 2006; Hayman and Thayer, 2009). These measurement errors result in cloudphase misidentification, which, in turn, introduce unquantified errors into observationally based understanding of key cloud and radiative processes. Observations by lidar of Arctic liquid-only and mixed-phase clouds in particular are challenging due to their high optical thicknesses, relative to iceonly clouds, and low-lying altitude, which demands large system dynamic ranges.

This work focuses on novel polarimetric lidar measurements made at Summit, Greenland $\left(72^{\circ} 35^{\prime} 46.4^{\prime \prime} \mathrm{N}\right.$, $38^{\circ} 25^{\prime} 19.1^{\prime \prime} \mathrm{W} ; 3212 \mathrm{~m}$ a.m.s.l.), as part of the Integrated Characterization of Energy, Clouds, Atmospheric State, and Precipitation at Summit (ICECAPS) program outlined by Shupe et al. (2013). The primary measurements to be presented are taken from the Clouds Aerosol Polarization and Backscatter Lidar (CAPABL), which was originally designed to measure polarization properties of clouds with emphasis on identifying horizontally oriented ice crystals (HOICs) and cloud phase (Neely et al., 2013). Analysis of 7 years of polarimetric lidar data observed by CAPABL has highlighted several uncertainties and biases that can cause errors in the interpretation of geophysical retrievals of cloud phase, primarily caused by systemic limitations to adequately observe the dynamic range in backscattered signals from clouds.

The outline of this paper is as follows. The measurement theory, upon which the retrievals within CAPABL's automatic processing are based, is given in Sect. 2. An overview of the data collection and processing is provided in Sect. 3 with emphasis on geophysical retrievals and potential errors caused by limited signal dynamic range. Several retrieval methods are presented and combined into a bestestimate cloud identification in Sect. 4. A comparison of the best-estimate data product is presented in Sect. 5 using a co-located micro-pulse lidar (MPL), microwave radiometer 
(MWR), millimeter cloud radar (MMCR), and broadband radiation measurement suite. Finally, this paper concludes with a discussion in Sect. 6 describing applicability of the presented observational methodology to other polar lidar measurements and quantification of lidar classification errors on radiation budget estimates.

\section{Measurement theory}

Observed polarization properties are a function of atmospheric scattering, optical system setup, and recording systems. For example, traditional two-channel polarization systems can not unambiguously measure atmospheric depolarization without additional information. Separating atmospheric depolarization from systematic effects is non-trivial. Alvarez et al. (2006) show, for example, how to calibrate differential detector sensitivity and receiver cross-talk, while Hayman and Thayer (2009) show how to remove depolarization effects caused by receiver optical retardance and scattering. However, recording systems that are subject to saturation, or underrepresentation of signal strength compared to incident irradiance, can also cause depolarization ratio effects, which are not constant in range and can not be calibrated using methods like that presented in Alvarez et al. (2006) or Hayman and Thayer (2009). Depolarization effects related to saturation link polarization measurements (microphysical properties) with properties like cloud base height, range, and optical thickness (macrophysical properties) that have a strong influence on the signal intensity of the measurements. Given the tight link between macro- and microphysical properties, optical system setup, and recording systems, adding more polarization measurements to the traditional two polarization lidar systems can greatly enhance the utility of lidar polarization measurements. The cost of this additional utility is the added formalism needed to represent the vector nature of light.

\subsection{Polarization measurements and Mueller formalism}

Using a vector description of light allows one to describe scatterers by how they alter polarization states of light as well as how much energy is redirected. Hayman and Thayer (2012) use polar decomposition of Mueller matrices to define the Stokes vector lidar equation (SVLE), which links transmitted and received polarization states of light to physical attributes of the scatterers. This equation forms the basis of CAPABL's polarization retrievals and is given in Eq. (1)

$$
\begin{array}{r}
\boldsymbol{N}(R)=\mathbf{O M}_{R_{x}}\left(\boldsymbol{k}_{\mathrm{s}}\right)\left[\left(G(R) \frac{A}{R^{2}} \Delta R\right) \mathbf{T}_{\mathrm{atm}}\left(\boldsymbol{k}_{\mathrm{s}}, R\right)\right. \\
\left.\mathbf{F}\left(\boldsymbol{k}_{\mathrm{i}}, \boldsymbol{k}_{\mathrm{s}}, R\right) \mathbf{T}_{\mathrm{atm}}\left(\boldsymbol{k}_{\mathrm{i}}, R\right) \mathbf{M}_{T_{x}}\left(\boldsymbol{k}_{\mathrm{i}}\right) \boldsymbol{S}_{T_{x}}+\boldsymbol{S}_{\mathrm{B}}\left(\lambda_{R_{x}}\right)\right],
\end{array}
$$

where $\boldsymbol{N}$ is vector of photon counts for each polarization channel as a function of range, $R ; \mathbf{O}$ is the observation matrix describing each polarization observation channel; $\mathbf{M}_{T_{x}}$ and $\mathbf{M}_{R_{x}}$ are the Mueller matrices describing the transmitter and receiver, which are functions of the incident and scattered wave vector $\boldsymbol{k}_{\mathrm{i}}$ and $\boldsymbol{k}_{\mathrm{s}}$, respectively; $G$ is the physical overlap function of the transmitter and receiver; $A$ is the telescope area; $\Delta R$ is the range resolution of the counting system; $\mathbf{T}_{\mathrm{atm}}$ is the one way transmission Mueller matrix either between the transmitter and the scatterer or between the scatterer and the receiver; $\mathbf{F}$ is the scattering phase matrix, which is a function of both transmitted and received wave vectors and range; $\boldsymbol{S}_{T_{x}}$ is the Stokes vector of the light from the laser source; and $S_{\mathrm{B}}$ is the Stokes vector of the background condition, which is a function of the receiver wavelength window, $\lambda_{R_{x}}$. The terms of the equation are organized by their functional order because matrix operations do not generally commute. The observation matrix is also included because only intensity can be measured directly with the full Stokes vector determined through measurement with particular configurations of the analyzer (Hayman and Thayer, 2012). For more information on the SVLE and its derivation, the reader is referred to Hayman and Thayer (2012).

Elements of $\mathbf{F}$ can be used to describe physical attributes of scatterers beyond simple scattering cross section (Van De Hulst, 1957; Mishchenko and Hovenier, 1995; Kaul et al., 2004). The reader is referred to Neely et al. (2013), who describe the polarization retrievals and the physical interpretation of the elements CAPABL measures in detail. The retrieval presented by Neely et al. (2013) is generalized here by relaxing the assumption made in that work that the receiver orientations (linear polarization angles), here given the variable name $\theta_{\mathrm{i}}$, are fixed at $\theta_{1}=0^{\circ}, \theta_{2}=45^{\circ}$, and $\theta_{3}=90^{\circ}$ relative to the output linear polarization. A full derivation of this generalization is given in Appendix A. The results are given here without further comment.

Volume depolarization, hereafter referred to as depolarization,

$d\left(R, \theta_{i}\right)-1=\frac{F_{33}\left(R, \theta_{i}\right)}{F_{11}\left(R, \theta_{i}\right)}=$

$\underline{\left(\cos \left(2 \theta_{3}\right)-\cos \left(2 \theta_{2}\right)\right) N_{1}(R)+\left(\cos \left(2 \theta_{1}\right)-\cos \left(2 \theta_{3}\right)\right) N_{2}(R)+\left(\cos \left(2 \theta_{2}\right)-\cos \left(2 \theta_{1}\right)\right) N_{3}(R)}$ $\sin \left(2 \theta_{2}-2 \theta_{3}\right) N_{1}(R)+\sin \left(2 \theta_{3}-2 \theta_{1}\right) N_{2}(R)+\sin \left(2 \theta_{1}-2 \theta_{2}\right) N_{3}(R)$

and volume diattenuation, hereafter referred to as diattenuation,

$D\left(R, \theta_{i}\right)=\frac{F_{12}\left(R, \theta_{i}\right)}{F_{11}\left(R, \theta_{i}\right)}=$

$\frac{\left(\sin \left(2 \theta_{3}\right)-\sin \left(2 \theta_{2}\right)\right) N_{1}(R)+\left(\sin \left(2 \theta_{1}\right)-\sin \left(2 \theta_{3}\right)\right) N_{2}(R)+\left(\sin \left(2 \theta_{2}\right)-\sin \left(2 \theta_{1}\right)\right) N_{3}(R)}{\sin \left(2 \theta_{2}-2 \theta_{3}\right) N_{1}(R)+\sin \left(2 \theta_{3}-2 \theta_{1}\right) N_{2}(R)+\sin \left(2 \theta_{1}-2 \theta_{2}\right) N_{3}(R)}$,

can be expressed in terms of arbitrary observation angles, $\theta_{i}$, assuming the condition $\zeta \neq 0 . \zeta$, defined as

$$
\begin{aligned}
\zeta= & \cos \left(2 \theta_{3}\right)\left(\sin \left(2 \theta_{2}\right)-\sin \left(2 \theta_{1}\right)\right)+\cos \left(2 \theta_{1}\right)\left(\sin \left(2 \theta_{3}\right)\right. \\
& \left.-\sin \left(2 \theta_{2}\right)\right)+\cos \left(2 \theta_{2}\right)\left(\sin \left(2 \theta_{1}\right)-\sin \left(2 \theta_{3}\right)\right),
\end{aligned}
$$

is the common denominator of a fraction that results from the inversion procedure described in Appendix A. For CA$\mathrm{PABL} \zeta \approx-2$ calculated from receiver polarizations via atmospheric calibration performed for each measurement. 
The expressions given in Eqs. (2) and (3) are generalizations of the equations presented by Neely et al. (2013) that assume fixed orthogonal receiver polarization angles. The diattenuation equations presented by Neely et al. (2013) in their Eqs. (7) and (20) can be recovered from our Eq. (3) by using $\theta_{1}=45^{\circ}, \theta_{2}=-45^{\circ}$, and $\theta_{3}=0^{\circ}$ for their Eq. 7 and $\theta_{1}=45^{\circ}, \theta_{2}=-45^{\circ}$, and $\theta_{3}= \pm 90^{\circ}$ for their Eq. (20). The depolarization term presented by Neely et al. (2013) in their Eq. (8) can be recovered with either set of angles from our Eq. (2). For clarity, retrievals performed with equations from Neely et al. (2013) are referred to as traditional or orthogonal as the polarizations used are orthogonal in Poincaré space. The retrievals using Eqs. (2) and (3) are referred to as non-orthogonal as they require no such assumption.

\subsection{Retrieval assumptions}

By assuming the more general form of the backscattering phase matrix, Eq. (A3), which allows for horizontal orientation of scatterers as opposed to only random orientation, and observing scatterers in an off-zenith direction (for CAPABL the tilt angle from zenith is $32^{\circ}$ ), no ambiguity arises in the interpretation of depolarization measurements as seen for example by Thomas et al. (1990) or Winker et al. (2009) where low depolarization, typically associated with liquid, from ice is observed from organized specular reflections off HOICs. Equations (2) and (3) are valid for randomly or horizontally oriented axially symmetric scatterers. If randomly orientated ice crystals (ROICs) are observed, diattenuation will be strictly $D=0$ and the scattering Mueller matrix simplifies to a function of two elements, depolarization $d$ and the volume backscatter coefficient $\beta$ (Hayman and Thayer, 2012). This form of the backscattering phase matrix is consistent with the works of Mishchenko and Hovenier (1995), Flynn et al. (2007), Gimmestad (2008), Hayman and Thayer (2009), and Hayman and Thayer (2012).

Traditional volume depolarization ratio, hereafter referred to as depolarization ratio, measurements are made by assuming random orientation of particles and using only two measurements of the polarization of the backscattered signal, one that is linear and parallel to the outgoing laser polarization and one that is linear and perpendicular to the outgoing laser polarization, e.g., Schotland et al. (1971), Sassen (1991), Mishchenko and Hovenier (1995), Gimmestad (2008), and Hayman and Thayer (2012). Depolarization, $d$, and depolarization ratio, $\delta$, can be related but are not equivalent. Depolarization is an element of the Mueller formalism and can be measured with any set of two polarizations (assuming randomly oriented particles), and the depolarization ratio is often related to the phase of atmospheric scatterers but is only measured with parallel and perpendicular polarizations. They are related as

$\delta(R)=\frac{N_{0_{\perp}}(R)}{N_{0_{\|}}(R)}=\frac{d(R)}{2-d(R)}$, where $N_{0_{\perp}}$ is the number of photons (or equivalently the photon arrival rate) at the detector surface in the perpendicular channel as a function of range, and $N_{0_{\|}}$is the number of photons (or equivalently the photon arrival rate) at the detector surface in the parallel channel as a function of range. Measuring orthogonal polarizations imposes a stringent requirement on a lidar system that can be lessened by using the more general form given in Eq. (2).

Implicit in the development of the SVLE, and most lidar retrievals, is the assumption that the observed signal is linearly related to irradiance of light at the receiver. For targets with low depolarization ratios like liquid and clear air, the signal dynamic range in the parallel and perpendicular channels can be dramatically different. A depolarization ratio of $1 \%$ would indicate the two signals would be different by 2 orders of magnitude, whereas a depolarization ratio of $50 \%$ would indicate the two signals would be different by a factor of 2. This difference is of practical concern as most observing systems have limited dynamic range, on the order of 4 to 5 orders of magnitude.

Finally, Eqs. (2) and (3) are also derived on the strict assumption that the lidar system emits a linear polarization and measures only linear polarizations (displaying no systematic retardance for example). These assumptions have been questioned for some optical systems (e.g., Hayman and Thayer, 2009, or Di et al., 2016) but have been directly measured for CAPABL. CAPABL has a transmitter polarization purity of $123: 1$ and a receiver polarization purity of $>800: 1$, resulting in a system bias in the depolarization ratio no greater than $0.8 \%$.

\subsection{Diattenuation}

The CAPABL system requires at least three polarization measurements to retrieve $F_{11}(R), F_{12}(R)$, and $F_{33}(R)$. However, saturation has been observed to cause biases in CAPABL measurements using only three polarizations, i.e., inability to measure all three signals over the entire dynamic range leading to an underrepresentation of signal strengths and causing biases in polarization retrievals. Thus, a fourth polarization channel is included, three to measure atmospheric properties and one to monitor recording system effects. For CAPABL, the $F_{12}(R)$ term is measured twice using two sets of polarization channels with opposite sensitivity to saturation, i.e., one set of measurements is biased in the positive direction by saturation while the other set of measurements is biased in the negative direction. If the $F_{12}(R)$ terms measured in two different ways are consistent at a given altitude, the lidar counting system is operating in a linear manner. An advantage of this over-constrained polarization retrieval is that CAPABL can actively monitor if the polarization measurements are acting properly or are causing systematic biases. A combination of any three of the four polarization channels can be used to optimize CAPABL's retrievals if the polarization signals are not subject to satura- 
tion. If $F_{12}(R)$ is zero, i.e no HOICs are present or the system is insensitive to orientation ${ }^{3}$, only two of the four channels are needed for atmospheric properties. However, if the polarization retrievals are subject to saturation, CAPABL's additional channels can be used to identify measurements with non-physical retrieved values and separate them from geophysical values. Therefore, including an extra polarization measurement and retrieving diattenuation can be used to verify two major assumptions: the presence of strictly ROICs and counting system linearity.

\section{CAPABL hardware, data analysis, and classification}

The theory described in Sect. 2 is, in principle, valid for any measurement system type and polarization angle selection. However, as a practical matter, limitations in measurement systems must be considered. Measurement system sensitivity and dynamic range are the main concern for this work and, in particular, the limited observational dynamic range of signals.

Broadly, lidar counting systems can be classified as either photon counting systems or as analog systems. Photon counting systems are capable of measuring weak light signals, which allow them to observe high altitudes effectively (relative to analog detection assuming ground-based measurements). Analog systems sacrifice sensitivity to measure stronger signals, which facilitates measurement of low altitudes. In photon counting, detector signals are discriminated with a fixed voltage threshold. This threshold is set to remove much of the electrical noise resulting from using single-photon, high-gain detectors. When a voltage signal is observed in excess of the threshold, a photo-electron is counted and its time of flight is assigned to a particular time bin. The intensity is presumed to be linearly related to the total number of counts in that bin over some integration period. Error can arise with this technique; however, if photons arrive at the counting system in close succession (Whiteman et al., 1992; Donovan et al., 1993). It is possible that pulses can pile up in such a way that two or more pulses either overlap in time or pass through the system faster than the counting system can reset itself. In either case, the intensity observed by the optical system is not linearly proportional to the number of photo-electrons counted because some photo-electrons have not been counted. In analog detection, the discrimination threshold is removed and the volt-

\footnotetext{
${ }^{3}$ Sensitivity to orientation is a combination of diattenuation per scatterer and backscattering efficiency of each scatterer. Measurements of diattenuation are a weighted average of the scatterers in the volume. CAPABL achieves sensitivity to HOICs through enhanced scattering near its tilt angle of $32^{\circ}$ with enhanced backscattering from corner reflections within crystals allowing for low diattenuation cases and minor subpopulations to be observed. Insensitivity to orientation is a result of the randomly oriented population of scatterers dominating the return signal.
}

age produced by the detector is passed through an analogto-digital converter with its amplitude providing the relative intensity of the collected backscattered signal. This method requires much higher signal-to-noise ratio than photon counting because it lacks a discriminator that separates the influence of detector circuit electrical noise from the desired signal.

\subsection{CAPABL hardware}

The CAPABL system has been deployed to Summit, Greenland, within the ICECAPS sensor suite since 2010 (Shupe et al., 2013; Neely et al., 2013). Since its installation several hardware modifications, completed in June 2015, have improved the system's overall observational capacity. These modifications are described with an emphasis on how they allow the CAPABL system to better observe clouds via enhancement of counting system dynamic range. The current system specifications are given in Table 1, which can be compared to Table 1 from Neely et al. (2013) for reference.

After several years of data collection, the original Nd:YLF laser was replaced by a more powerful Nd:YAG laser. This changed the laser wavelength from 523 to $532 \mathrm{~nm}$. The optical components were changed accordingly. In addition, the original $35.6 \mathrm{~cm}$ telescope was replaced by a smaller $20.8 \mathrm{~cm}$ Schmidt-Cassegrain telescope to allow the system to be more easily tilted; the current tilt angle, set in June 2015 , is $32^{\circ}$ from zenith. The photo-multiplier tube (PMT) was upgraded from the original PMT, a Thorn EMI 9863B/100, to a Hamamatsu R7400U-03. These modifications have enhanced the power aperture product and the detection sensitivity of the system, which increased the overall signal-to-noise ratio.

The second major change was an upgrade of the receiver counting system from a purely photon counting system to a combined analog and photon counting system. By using a counting system that combines photon counting and analog detection, saturation in photon counting caused by high count rates is ameliorated by using analog detection, approximately $>10 \mathrm{MHz}$, while maintaining sensitivity to low count rates, approximately $<1 \mathrm{MHz}$, using photon counting detection. More about this counting system can be found in Newsom et al. (2009).

CAPABL observes four non-orthogonal receiver polarization channels. These polarizations are all linear and were oriented parallel to the outgoing polarization, $0^{\circ}$, perpendicular to the outgoing polarization, $90^{\circ}$, approximately $30^{\circ}$ from parallel (or $60^{\circ}$ from perpendicular) polarization (referred to as third channel), and approximately $110^{\circ}$ from parallel (or $20^{\circ}$ from perpendicular) polarization (referred to as fourth channel). Combining the new counting system and the four linear polarizations with the non-orthogonal polarization theory, this allows eight methods to solve Eqs. (2) and (3). This variety of inversion methods grants flexibility to optimize polarization measurements. 
Table 1. CAPABL current system specifications. Polarization purity and polarization rejection are measured quantities. Polarization purity is measured with a $100000: 1$ Glan-Taylor polarizer.

\begin{tabular}{|c|c|c|}
\hline Transmitter & Receiver & Signal processing \\
\hline $\begin{array}{l}\text { Big Sky Laser Ultra flashlamp } \\
\text { pumped Nd:YAG }\end{array}$ & Schmidt-Cassegrain telescope & $\begin{array}{l}\text { Combined analog and photon } \\
\text { counting acquisition }\end{array}$ \\
\hline Wavelength: $532.3 \mathrm{~nm}$ & Receiver aperture: $20.8 \mathrm{~cm}$ & Data system: \\
\hline Pulse energy: $60 \mathrm{~mJ}$ & Filter bandwidth: $0.3 \mathrm{~nm}$ & Licel transient recorder TR20 12 bit \\
\hline Pulse rate: $15 \mathrm{~Hz}$ & Channels: 1 & Range bin size: $7.5 \mathrm{~m}$ \\
\hline Twin head & Field of view: $1.4 \mathrm{mrad}$ & Integration time: $5 \mathrm{~s}$ \\
\hline Polarization purity: $>123: 1$ & $\begin{array}{l}\text { Polarization rejection: }>800: 1 \\
\text { Linear polarizations observed: } 4\end{array}$ & PMT: Hamamatsu R7400U-03 \\
\hline
\end{tabular}

Table 2. A summary of the data processing steps taken to create the data masks desired for CAPABL. The processing for each data type - analog (An), photon counting (PC), and saturation-corrected photon counting (SCPC) - is constant except where noted. Note that the depolarization and diattenuation error equation are calculated per standard propagation of error techniques taking a Taylor series expansion of Eqs. (2) and (3).

\begin{tabular}{|c|c|c|}
\hline & Processing Step & Details \\
\hline (1) & Time integration & To a constant $20 \mathrm{~s}$ resolution \\
\hline (2) & Spatial integration & To a constant $30 \mathrm{~m}$ resolution \\
\hline (3) & Saturation correction (PC data) & Creates SCPC level \\
\hline (4) & Background subtraction & \\
\hline (5) & SNR filter & \\
\hline (6) & Speckle filter & $\begin{array}{l}5 \times 5 \text { surrounding box } \\
>75 \% \text { data already removed }=\text { bad } \\
>25 \% \text { data available }=\text { good }\end{array}$ \\
\hline (7) & Calculate polarization properties & $\begin{array}{l}\text { Depolarization and depolarization ratio per Eqs. (2) and (5) } \\
\text { Depolarization and depolarization ratio error per error } \\
\text { propagation of Eqs. }(2) \text { and }(5) \\
\text { Diattenuation per Eq. (3) } \\
\text { Diattenuation error per error propagation of Eq. (3) } \\
\text { Backscatter ratio }(R) \text { per Klett (1981), Neely et al. (2013) }\end{array}$ \\
\hline (8) & Remove non-physical values & $\begin{array}{l}\text { Values outside } 0 \leq \delta_{O} \leq 1 \\
\text { Values outside } 0 \leq \sigma_{\delta_{O}} \leq 0.4 \\
\text { Values outside }-1 \leq D \leq 1 \\
\text { Values outside } 0 \leq \sigma_{D} \leq 0.2\end{array}$ \\
\hline (9) & Calculate base mask & $\begin{array}{l}\text { Clear: } 1 \leq R<2.6 \\
\text { Aerosol: } 2.6 \leq R<6.5 \\
\text { Cloud: } R \geq 6.5\end{array}$ \\
\hline (10) & Calculate phase mask & $\begin{array}{l}\text { Liquid: cloud voxels with } 0 \leq \delta_{O} \leq 0.11 \\
\text { Ice: cloud voxels with } \delta_{O}>0.11\end{array}$ \\
\hline (11) & Calculate orientation mask & $\begin{array}{l}\text { Random: ice with } 0 \leq D_{1} D_{2} \leq 0.01 \\
\text { Preferential: ice with } D_{1} D_{2} \geq 0.01 \text { and } \sigma_{D} \leq 0.05 \\
\text { Saturation: ice with } D_{1} D_{2} \leq-0.01\end{array}$ \\
\hline
\end{tabular}

\subsection{Processing methods}

Data analysis and classification is performed by taking advantage of CAPABL's variety of polarization signal measurements. There are several levels of processing and filtering to ensure data quality. These are implemented in an automatic algorithm. The steps are given in Table 2 and described here in order.
CAPABL makes observations with $5 \mathrm{~s}$ resolution per polarization angle and scans through four polarization angles before returning to the original polarization, taking a total of $20 \mathrm{~s}$ before returning to the first polarization angle. The outgoing polarization is $45^{\circ}$. These data are time-integrated to $20 \mathrm{~s}$ per polarization and spatially integrated to the resolution of $30 \mathrm{~m}$. Non-paralyzable saturation corrections are applied per the method described in Appendix B and by Whiteman (2003) to the photon counting data. Note that the vari- 
ance of saturation-corrected photon counting is not simply the variance from Poisson statistics but also the error introduced by an inexact model fit is taken into account for all error analyses and is described in Appendix B. All data are then background-subtracted and subject to an SNR filter. Photon counting data with less than one photon count per bin after background subtraction and analog voltages less than $1 \mathrm{mV}$ per bin after background subtraction (SNR ratio of approximately $-8 \mathrm{~dB}$ ) are removed. These background-subtracted and SNR-filtered data are then passed through a speckle filter, which interrogates a 5 by 5 time and altitude bin region, referred to here as a voxel (volume pixel), around each voxel of interest. Voxels, where more than $75 \%$ of the surrounding data are removed by the SNR filter, are also removed.

Depolarization, depolarization ratio, and diattenuation as well as their error estimates are calculated using the orthogonal polarization approach presented by Neely et al. (2013), and also using the non-orthogonal approach described here. The orthogonal approach uses all the same steps as Neely et al. (2013) but with the following exception. Instead of assuming the observations are made at exactly (1) parallel, $0^{\circ}$; (2) perpendicular, $90^{\circ}$; and (3) $45^{\circ}$, the angle of the third channel is carried through the analysis as a variable and the retrieved angle from atmospheric calibration is used. This is designed to accommodate for slight retardance changes in the liquid crystal variable retarder (LCVR) as a function of ambient laboratory temperature. For the depolarization retrieval in areas that lack oriented scatterers, the depolarization can be calculated with any two of the receiver polarization channels. HOICs are identified by non-zero diattenuation, $D$. Diattenuation is calculated in two ways, (1) using parallel, perpendicular, and the third channel referred to as $D_{1}$ and (2) using parallel, perpendicular, and the fourth channel referred to as $D_{2}$. These channels are chosen because of their opposite sensitivity to saturation for the photon counting and saturation-corrected photon counting retrievals. By multiplying the two measurements together, negative values indicate $D_{1}$ and $D_{2}$ are tending in opposite directions indicating a saturation event. Conversely, positive values of $D_{1} D_{2}$ indicate the two measurements are tending together and that the nonzero diattenuation is physical, i.e., unaffected by saturation.

Data are removed outside of the allowable ranges, $0 \leq d \leq$ $1,0 \leq \sigma_{d} \leq 0.4,-1 \leq D \leq 1$, and $0 \leq \sigma_{D} \leq 0.2$, as these represent non-physical conditions. Note that the error analysis procedure for photon counting described by Neely et al. (2013) assumes Poisson statistics where the data are assumed shot noise limited. The same procedure for photon counting is carried through the analysis shown here. The analog signal is not governed by Poisson statistics, however. The analog uses the variance of the background voltages for its error estimates. Additionally, as mentioned above, the variance for saturation-corrected photon counting is modified to reflect the correction procedure and the variance introduced via inexact model fitting. Finally the backscattering ratio, the ratio of total scattering to molecular scattering, is calculated. Ex- pected molecular scattering is calculated using temperature and pressure information collected from the ICECAPS twice daily radiosonde program, interpolating between launches. The inversion technique of Klett (1981) is used to calculate total scattering coefficient as described by Neely et al. (2013). A lidar ratio of 10 is assumed, following the results of Hoffmann et al. (2009) and review of Nott and Duck (2011) and references therein, to convert the total extinction derived by the Klett inversion to total backscattering coefficient.

The thresholds set for the automated classification algorithm are important to the interpretation of the results of this work. Depolarization and diattenuation are both elements of Mueller matrices, which are defined to have absolute values less than or equal to unity. Values outside this are nonphysical. The values on the depolarization and diattenuation error bounds are limited mostly by background irradiance, which is tuned via receiver hardware. A receiver neutral density filter lowers both the signal count rates and atmospheric background count rate by a factor of 1000 , which brings the majority of the signal intensity into the desired dynamic range of the counting system and makes the depolarization and diattenuation error values limited only by shot noise. The filters, which remove data points based on depolarization and diattenuation and their errors, remove less than $3 \%$ of all data values. For context, background and speckle filters remove approximately 60 and $23 \%$, respectively, of all data points.

By design, CAPABL uses four polarization channels to measure three elements of the scattering Mueller matrix: $F_{11}, F_{12}$, and $F_{33}$ with one additional measurement to monitor saturation. If saturation is not an issue, any three of the four channels may be used for the inversion of polarization properties. Thus, the utility of the generalization given in Sect. 2 is that the three signals with the least error can be used at any time. For example, the three strongest signals for measurements of high ice clouds where backscattered signals are weaker or the three weakest measurements for low liquid clouds where the backscattered signal is stronger. Using non-orthogonal polarizations allows the dynamic range between polarization components to be accommodated and optimized.

\subsection{Classification}

Using all of the polarization processing listed above, the classification of data is performed in the following manner. Clear air is found as any voxel with a backscattering ratio less than 2.6. Sub-visible clouds and aerosols are any voxel with a backscattering ratio between 2.6 and 6.5. Clouds are tagged as voxels with backscattering ratio greater than 6.5 . For reference, Cesana and Chepfer (2013) use a threshold value for backscattering ratio of 5 to identify cloudy scenes. Within cloud voxels, the depolarization ratio threshold, originally defined by Intrieri et al. (2002) of $\delta_{O} \geq 0.11$ was used to define ice and $\delta_{O}<0.11$ as water. Any voxels tagged as aerosol 
that displays a depolarization ratio $\delta_{O} \geq 0.11$ is reset as ice. HOICs are identified by $D_{1} D_{2}>0.01$ with $\sigma_{D_{1}}, \sigma_{D_{2}} \leq 0.05$.

Classified lidar profiles can then be condensed to a single column classification for the radiatively dominate species, referred to as the column type. If a column contains liquid voxels at any altitude, the column is labeled as liquid. If a column lacks liquid but contains ice voxels, it is labeled as ice. Ice is separated into two categories. If the column is labeled ice and contains HOICs at any altitude, it is labeled HOIC, or ROIC otherwise. If the column contains no liquid or ice but contains sub-visible voxels, it is labeled sub-visible. Finally, if the column lacks all other types of voxels, it is labeled as clear. One note is that this method can misclassify areas that lack lidar data as clear air. Lidar data can be lacking due to attenuation due to low-lying fog, clouds below lidar overlap, or an obstructed lidar window. In this case, data can be mistakenly classified as clear air. As a final check, data that are classified as clear air must have substantial signal around $2 \mathrm{~km}$ altitude. This requires more than $66 \%$ of data for a profile between 1 and $2 \mathrm{~km}$ passes all other filtering steps; otherwise it is tagged as obscured instead of clear air.

The setting of the backscatter ratio bounds is more subjective. As there is no true molecular measurement at Summit (for example provided by a Raman lidar or high-spectralresolution lidar), the Klett inversion was used assuming a lidar ratio of 10. Curry et al. (1996) note that clear air is uncommon in the Arctic. It has been the authors' experience that even the clearest days at Summit still have some amount of ice in the sky. The clearest day observed within May and June 2015 is used as a baseline to set the clear air threshold of 2.6. The threshold limits between aerosol or sub-visible clouds and clouds were set using an all-sky camera. The thinnest visible cloud layer observed during the same time period was used to separate the aerosol or sub-visible clouds and cloud classifications.

The threshold between liquid and ice, $\delta_{O}=0.11$, is taken from literature related to the Depolarization and Backscatter Unattended Lidar (DABUL) (Intrieri et al., 2002; Shupe and Intrieri, 2004; Zuidema et al., 2005; Shupe et al., 2006), which was the predecessor to CAPABL, and not changed for this work. An analysis was performed (not shown) of the effect of this threshold on cloud fractional occurrence (FO), the ratio of a particular single column classification type to all measurements. This analysis shows that thresholds between $\delta_{O}=0.11$ and $\delta_{O}=0.2$ change the FO of liquid and ice by less than $1 \%$ over the period of a month for July 2015 . Thresholds below $\delta_{O}=0.11$ significantly alter the FO of liquid and ice making $\delta_{O}=0.11$ a reasonable threshold value.

\subsection{Algorithm examples}

An example of this data classification procedure is given in Fig. 1 for analog detection and Fig. 2 for photon counting detection for 29 February 2016. This day is chosen because it contains both single-level and two-level mixed-phase cloud systems as well as high ice clouds. In comparing these two figures in the first $12 \mathrm{~h}$ of the day, the mixed-phase cloud layer at approximately $1.5 \mathrm{~km}$ altitude has been identified with substantially more liquid voxels when classified using analog detection than using photon counting detection. Furthermore, there are two smaller mixed-phase cloud layers that exist below $1 \mathrm{~km}$ between 03:00 and 05:00 and 08:00 to 11:00 UTC identified by analog detection, which are interpreted as purely ice when classified with photon counting observations. This discrepancy in interpretation is directly linked to cloud macrophysical properties, such as base height and optical depth that result in high count rates and cause saturation of the photon counting parallel channel. This increases the observed depolarization ratio by reducing the parallel photon count rate beyond the liquid-ice threshold and alters the derivative of the signal intensity that affects the Klett inversion.

To demonstrate that the day selected is not anomalous, monthly statistics are compiled for the first 4 months of data available, 2 July to 31 October 2015, since the hardware updates described. Over this time, the CAPABL system ran continuously and had an uptime of $>99 \%$ (this equates to approximately $5 \mathrm{~min}$ of missed data per day, which occurs at midnight UTC each day to perform system diagnostics and housekeeping). Voxels are separated by cloud phase and clear air. Voxels are integrated over a month-long period for each altitude and time bin. These data are compiled into box-andwhisker plots given in Fig. 3 for liquid voxels only (liquid, ice, and clear air voxels are given in Appendix C, Fig. C1). The median altitude of all liquid voxels is given as a line through the center of the box, which is completed by the 25th and 75 percentile of all monthly data. The whiskers extend to the 5th and 95th percentiles. The other data values are considered outliers.

Figure 3 indicates that median altitude of liquid voxels is not constant between analog, photon counting, and saturation-corrected photon counting (SCPC) for either orthogonal or non-orthogonal retrievals. There is a clear 1 to $2 \mathrm{~km}$ offset in the medians between analog and photon counting $(1.72,1.43,0.75$, and $0.91 \mathrm{~km}$ offsets for July, August, September, and October, respectively). This offset in mean voxel height indicates that low-level, liquid clouds are often misclassified by the photon counting channel as indicated by Figs. 1 and 2.

\section{Merged best-estimate cloud product}

The classification results of Figs. 1, 2, and 3 raise the question, "What retrieval technique is most accurate?". None of the results presented is perfect as each technique has innate benefits based on counting system dynamic range. For example, analog detection is designed for stronger signals, and photon counting detection for weaker signals. A single combination of all of the CAPABL data products leverages all of 


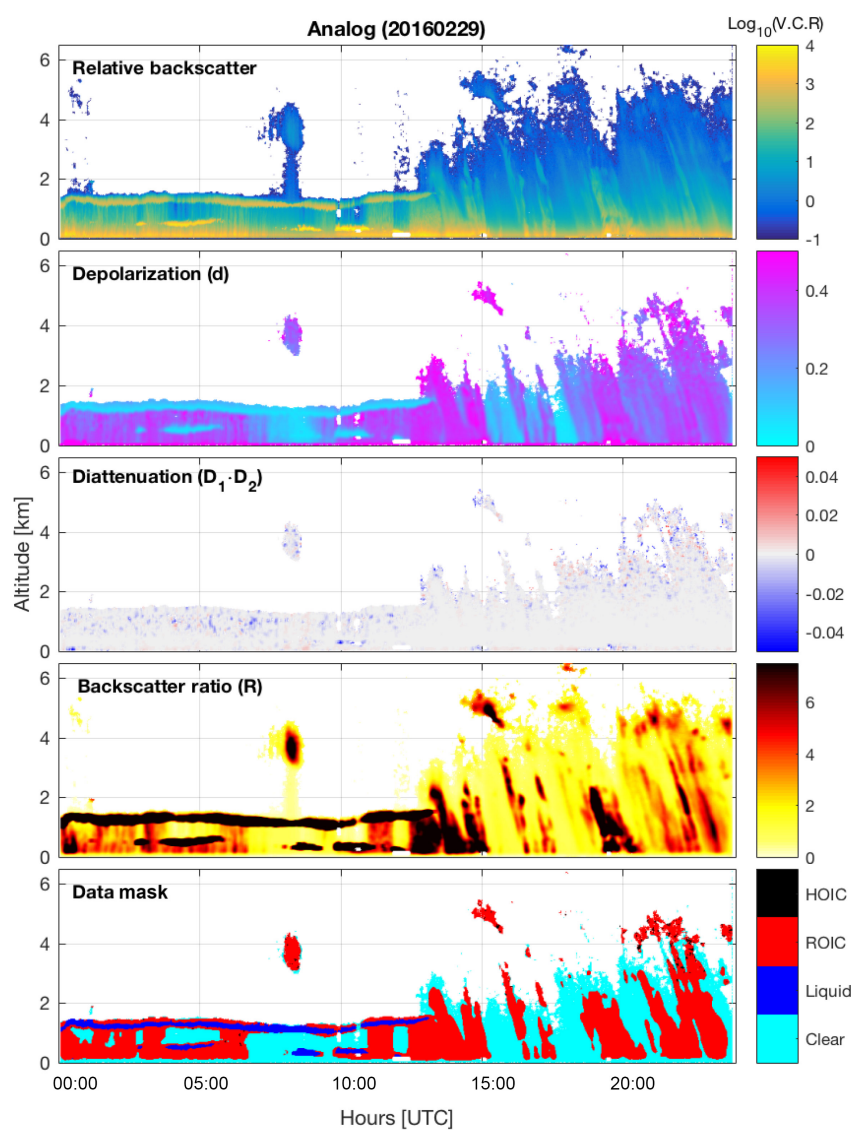

Figure 1. Analog data from the CAPABL system for 29 February 2016. Relative backscatter is the summation of backgroundsubtracted parallel and perpendicular voltages converted to a virtual count rate (V.C.R.) in MHz. Depolarization is calculated as given in Eq. (2). Diattenuation is calculated as given in Eq. (3) and multiplied to $D_{1} D_{2}$. Backscatter ratio is calculated by performing a Klett inversion and using ICECAPS radiosonde data (launched at 24:00 and 12:00 UTC daily) to calculate a molecular extinction component (Klett, 1981). The data mask given is calculated using rules described in Sect. 3.

the advantages of analog and photon counting observations as well as non-orthogonal polarization retrievals to extend the dynamic range of the counting system. This section describes the broad rules used to combine all of the possible data collected into a single best-estimate profile. This merging is done on the basis of signal counting regimes. Here valid signal ranges are defined where the measured signal count rate is linearly proportional to incident intensity at the detector. For analog detection, the range is fixed by the ana$\log$ noise in the detector circuit on the low end and by the width of the analog-to-digital converter (ADC) bounds on the high end. For photon counting, the range is fixed by the discriminator threshold and pulse height distribution on the low end and detector and counting system dead time on the high end.

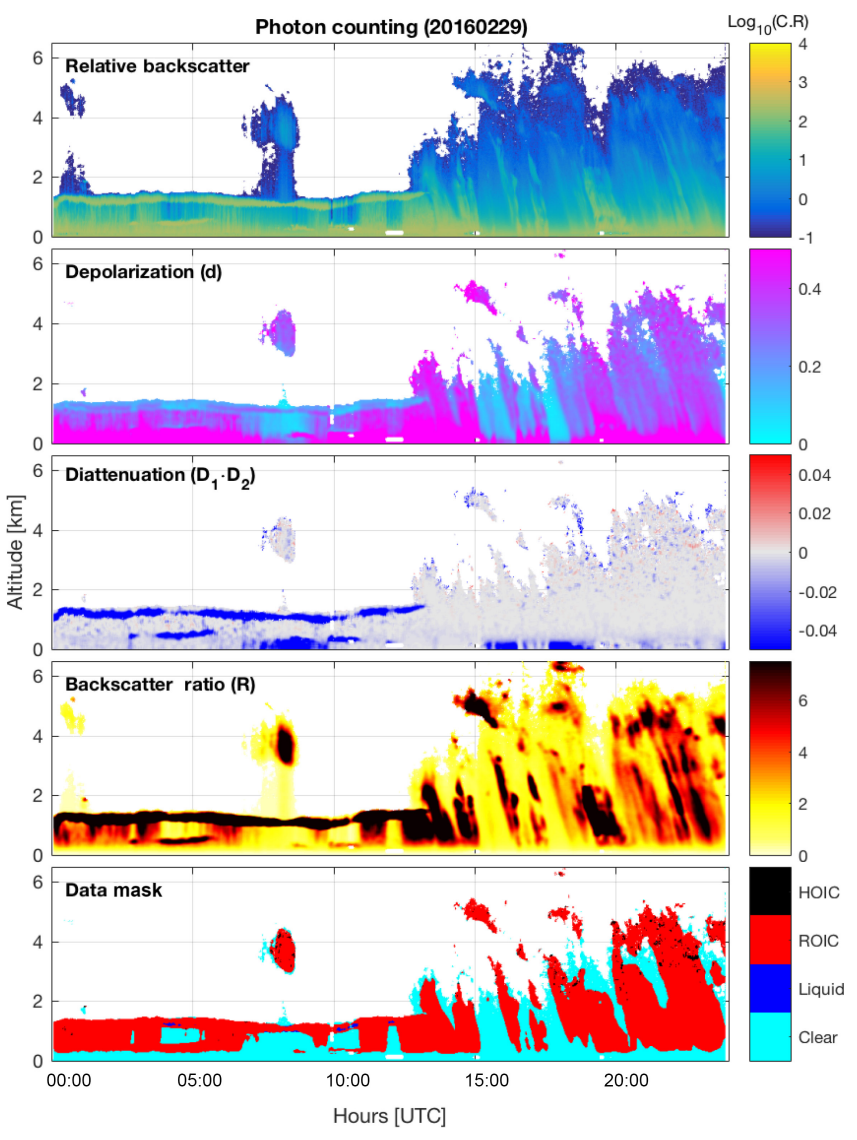

Figure 2. Same as Fig. 1 except photon counting data are shown.

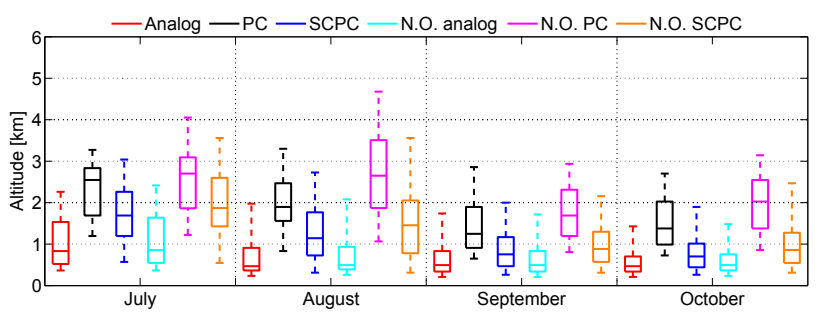

Figure 3. CAPABL binned liquid data from July 2015 to October 2015. The median is indicated by a line through the box, the 25 th to 75 th percentile ranges complete the box, and the whiskers extend to the 5th and 95th percentiles. PC, SCPC, and N.O. stand for photon counting, saturation-corrected photon counting, and nonorthogonal, respectively.

The SNR filter and the speckle filter are designed to remove data lacking signal strength in one or more of the polarization signals. These filters are applied to all data streams individually (to each polarization and counting type) and provide a lower limit of acceptable count rates for all channels. This limit is much higher for analog detection (approximately $1 \mathrm{MHz}$ ) and much lower for photon counting detection (approximately $10-100 \mathrm{kHz}$ ). The upper limit of count 
rate is enforced via bounds set on the receiver ADC. The ana$\log$ counting system is able to track PMT signals that exceed the ADC bounds. This occurs either with a PMT pulse that is too large or with multiple PMT pulses piling up in succession or with a pulse that has too large of a voltage rebound. The ADC bounds are set from -495 to $5 \mathrm{mV}$ with negative tending detector signals, which are nominally set to result in PMT pulses of approximately $10-15 \mathrm{mV}$. In all cases, if any shot results in any altitude bin signal on any polarization outside the valid ADC range, that altitude bin is removed from the data stream (hereafter referred to as clipping). Such clipped signals compose approximately $0.78 \%$ of all data from 0 to $8 \mathrm{~km}$ and are removed from both analog and photon counting detection data streams as they represent counting data that are no longer linearly proportional to incident intensity.

Applying the above filters to analog and photon counting raw data forces the data outside the valid counting range to be removed. For the analog signal, the data above the valid counting range are removed by the clipping filter, and the data below the valid count range are removed by the SNR and speckle filters. For the photon counting signal, the data below the valid count range are also removed by the SNR and speckle filters. The upper range of photon counting signal is, however, not necessarily limited by the clipping filter. In fact it is still poorly constrained due to possible pulse pileup. To specify the upper bound of the valid signal range for photon counting signals, the combination of analog and photon counting is considered. Implicit in the combined detection of analog and photon counting data is the assumption that there exists a range of counting signals, in the range of approximately $1-10 \mathrm{MHz}$, where both signals are acting linearly, i.e., that both measurement values reported are linearly proportional to the incident intensity at the detector. By this assumption, all data measured by the analog channel will be an upper bound on the photon counting detection. Practically speaking, this means that data removed from the analog detection scheme by the SNR and speckle filters are potentially valid photon counting data. Saturation-corrected photon counting is not needed.

All data types are processed as described in Sect. 3 removing all invalid signals. Data are stitched together by first taking all valid orthogonal analog signals. Any locations where valid orthogonal photon counting signals are present that are not previously covered by analog are then added. Nonorthogonal data using the three strongest signals for analog first then photon counting are then added where available. Non-orthogonal data using the three weakest signals for ana$\log$ only is then added to fill low altitude areas that may have been removed due to the parallel channel's clipping filter.

There exists another way of viewing analog, photon counting, and saturation-corrected photon counting data, which is presented by Newsom et al. (2009), referred to as gluing. This work will not perform the gluing procedure presented for several reasons: first it is impractical to calculate gluing coefficients for CAPABL by atmospheric calibration as ac- cess to the CAPABL system is limited to once or twice a year, second it is not clear how to combine analog and photon counting signals at a single height to adequately account for error introduced by temporal variation of gluing coefficients, third it is not clear how the range correlation of signals required for the Klett inversion method is affected by the thresholds of the gluing procedure, and finally, combining the data at the product level, and not the raw data level, is already required to combine orthogonal and non-orthogonal retrievals.

In contrast to these issues with data gluing, the method described above and used for this work addresses these problems in the following ways. Primarily, there is no need to track the temporal variation of gluing coefficients. By performing polarization retrievals as described, the time dependence of the detector is effectively canceled by ratio values of the polarization measurements. This method effectively reduces the assumption of a time variance in the detector from hours to the time it takes to make a complete polarization measurement set, which for CAPABL is $20 \mathrm{~s}$. Additionally, the range correlation required by the Klett inversion is preserved by considering each type of profile individually. Moreover, by systematically verifying each detector signal is within the counting system's observable and valid dynamic range, polarization retrievals can track Poisson or Gaussian errors (associated with photon counting and analog detection, respectively) in a more accountable way. Finally, as a practical matter, access to CAPABL occurs approximately once or twice per year. The method used allows the optical attenuation in the receiver to be set once and left untouched for the year.

An example of the merged data product is given in Fig. 4 for 22 August 2016. The raw analog signals are provided in the top panel, the merged data product in the middle panel, and the origin of the data for each voxel in the lower panel. This procedure takes most of its data from analog detection during daytime and low cloud scenes, much more data from photon counting during nighttime, and in the upper clear air and cloud scenes from non-orthogonal retrievals.

Considering the 4-month period of Fig. 3, monthly FO values are calculated by CAPABL from its column data classification. FO is calculated for all types of data processing as well as the best-estimate data product in Fig. 5. Figure 5 clearly illustrates several features. First, photon counting and saturation-corrected photon counting dramatically underestimate the occurrence of liquid clouds. This is because both methods have strong saturation-induced biases, linked to cloud base height and optical depth, which lower the observed parallel count rate artificially raising the observed depolarization and consequentially depolarization ratio. This serves to flip the classification of most water clouds to ice clouds. Second, analog detection underestimates overall cloud fraction due to its sensitivity - i.e., analog detection only sees clouds that are lower and more optically thick but misses many high tenuous clouds. Finally, in all cases, the 


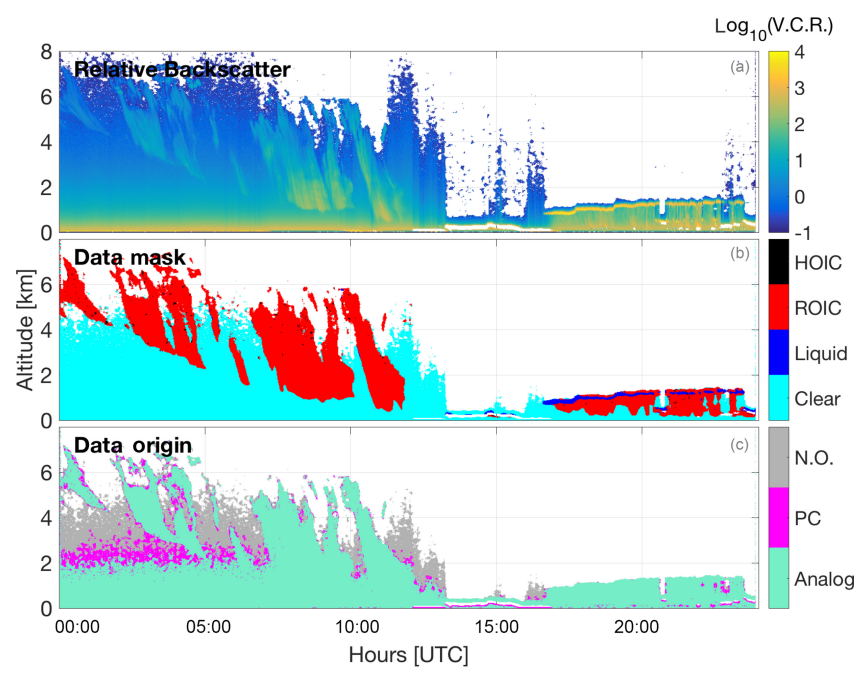

Figure 4. A sample of the CAPABL merged data product from 22 August 2016. Panel (a) shows total analog backscatter for the whole day in log base 10 signal intensity. Panel (b) shows the merged data product. Panel (c) shows the origin of each voxel. Analog indicates orthogonal processing with analog data, PC indicates orthogonal processing with photon counting data. All nonorthogonal types are lumped together as N.O.

merged data have less clear air than the single measurement techniques caused by an extended dynamic range and altitude range of observable signals.

\section{Multisensor comparison}

Comparison of remote sensing instrumentation that lack traceable calibration standards such as polarization lidars is of particular importance (Freudenthaler, 2016). This section evaluates the CAPABL cloud identification data product by using ancillary measurements taken by the ICECAPS program, namely a co-located micro-pulse lidar (MPL), millimeter cloud radar (MMCR), microwave radiometer (MWR) and broadband radiation measurements. The period of comparison is from July to December 2016. For this period, each sensor had an uptime of better than $95 \%$; one major reason for the period selected is the simultaneous measurement of much of the ICECAPS suite. This period also covers both polar day and night.

One important note for interpreting the results presented is the instrument pointing angle for CAPABL, MPL, MWR, MMCR, and radiation measurements. CAPABL operates at $32^{\circ}$ off zenith, the MPL at approximately $5^{\circ}$ off zenith, and the MMCR within $0.2^{\circ}$ of zenith. The radiation measurements are approximately $600 \mathrm{~m}$ away from CAPABL, MPL, MWR, and MMCR measurements and are total hemispheric measurements instead of narrow field of view. Given these constraints, the assumption of horizontal homogeneity of the scene above the site on the order of $500 \mathrm{~m}$ is required for an average voxel height of $2 \mathrm{~km}$.

\subsection{Co-located instruments}

\subsubsection{Micro-pulse lidar (MPL)}

The MPL used in this work is a Sigma Space V4 polarization sensitive system provided to the project by the ARM Program. The MPL uses a frequency-doubled Nd:YAG laser at $532 \mathrm{~nm}$. The system hardware design is well described by Campbell et al. (2002) and the polarization hardware and retrievals by Flynn et al. (2007).

MPL data are processed as follows. MPL raw data (photon counts) are time- and space-integrated as close as possible to CAPABL's data grid. Calibrations as described by Campbell et al. (2002) are performed monthly to remove signalinduced noise (SIN) resulting from the strong light signals from the shared telescope transceiver design. The SIN calibration corrections applied are linear interpolations between subsequent SIN calibrations. The calibration data are taken at $30 \mathrm{~m}$ resolution, which sets the lidar range resolution of this study. These SIN-corrected raw data are then linearly interpolated from the MPL grid directly to the CAPABL grid. The polarization properties are calculated as in Flynn et al. (2007) with no modification to the method presented. Note that the MPL measures depolarization using both linear and circular polarizations, while CAPABL measures only linear polarizations. A comparison of the specifications of CAPABL and the MPL is given in Table 3.

MPL data are classified as for CAPABL. Note that the MPL is only a photon counting system, while CAPABL uses both analog and photon counting, and CAPABL has the unique ability to measure the $F_{12}$ element of the scattering matrix upon which the diattenuation measurement is based. Filtering steps based on diattenuation and classification for HOICs are not performed by the MPL given the inability to make $F_{12}$ measurements. For this study, MPL data result in voxel classifications that are either clear air, cloud ice, cloud liquid, or removed due to data filtering.

\subsubsection{Millimeter cloud radar (MMCR)}

The MMCR used in this study was developed and provided by the National Oceanic and Atmospheric Administration's (NOAA) Earth Systems Research Laboratory. The MMCR is a $35 \mathrm{GHz}$ single-polarization Doppler radar. A general hardware description is given by Moran et al. (1998) and its software and operational measurement modes documented by Clothiaux et al. (1999). Data products available are based on observed Doppler spectra. Specifically, the system reports reflectivity (the integral of power in the Doppler spectrum), mean Doppler velocity (the first moment of the Doppler spectrum), and Doppler spectral width (the second moment of the Doppler spectrum). The zenith-pointing system occu- 

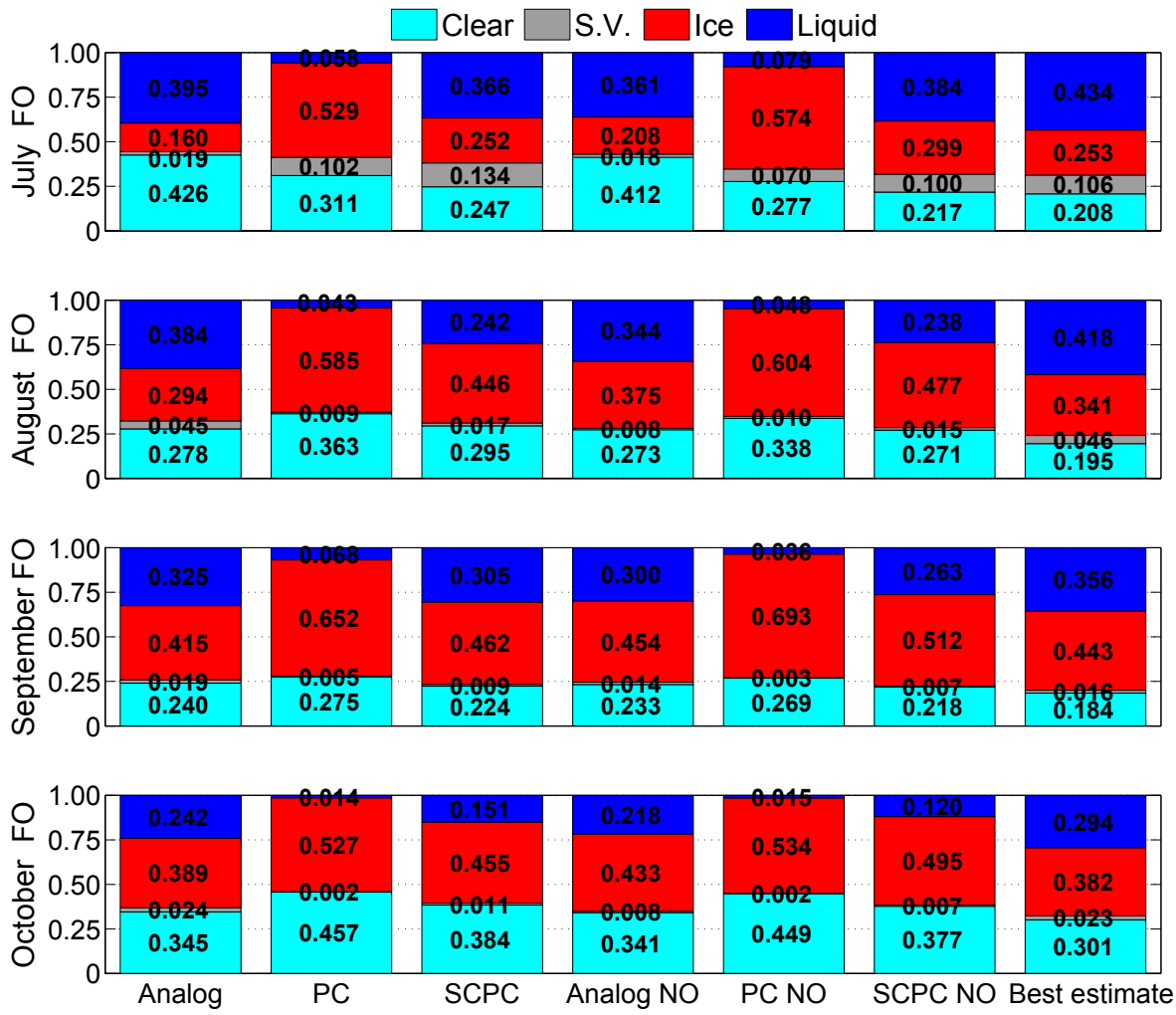

Figure 5. Fractional occurrence (FO) of each voxel type in the column for July 2015 to October 2015. To be labeled clear, the column must lack all sub-visible, ice, and water voxels. To be labeled sub-visible, the column must lack ice or water voxels. To be labeled as ice, a column must lack water voxels. If a column contains a water voxel, the column is labeled as liquid. The FO is given for each bar rounded to the nearest thousandth.

Table 3. Hardware comparison of relevant CAPABL and MP lidar specifications. The resolutions quoted are limited in range by the MPL afterpulse calibration data and in time by the CAPABL scan rate. The resolutions presented are as close as the data can be processed before linear interpolation of MPL data to CAPABL's data grid. Effective power aperture product is reduced for CAPABL by the receiver attenuation by a factor of 1000 .

\begin{tabular}{lrr}
\hline Specification & CAPABL & MPL \\
\hline Laser power (W) & 0.3 & 0.02 \\
Receiver attenuation (OD) & 3 & 0 \\
Telescope diameter (mm) & 208 & 178 \\
Effective power/aperture product $\left(\mathrm{W} \mathrm{mm}^{-2}\right)$ & 10.2 & 497 \\
Polarizations & 4 & 2 \\
Range resolution (m) & 25.98 & 30 \\
Polarization scan resolution (s) & $\approx 82$ & 80 \\
\hline
\end{tabular}

pies space in the same building as CAPABL and is carefully leveled by an on-site technician as needed to within approximately $0.2^{\circ}$ of zenith as the snow on which the building sits settles.

Data used for this study are from the radar general mode and high-sensitivity mode, referred to here as cirrus mode, with some operational settings given in Table 4. Radar data are generally taken at higher temporal resolution and lower spatial resolution than CAPABL. To represent the radar data onto a similar grid as CAPABL and the MPL, radar data are incoherently averaged in time to as close to the CAPABL grid as possible. Then, as with the MPL, data are linearly interpolated in time and space to the CAPABL grid.

\subsubsection{Microwave radiometer (MWR)}

Column moisture measurements are calculated using two colocated MWRs manufactured by Radiometer Physics GmbH (RPG). The first radiometer, an RPG humidity and temperature profiler (HATPRO), samples 14 channels from 22.2 to $60 \mathrm{GHz}$, of which 23.8 and $31.4 \mathrm{GHz}$ are used to retrieve precipitable water vapor and cloud liquid water, while the second radiometer, an RPG LWP-90-150, samples at 90 and $150 \mathrm{GHz}$. From microwave brightness temperature observations, the column liquid water path (LWP) is retrieved using physical retrievals and an optimal estimation algorithm. The LWP uncertainty using the $23.8,31.4,90$, and $150 \mathrm{GHz}$ data in the retrieval is approximately $5 \mathrm{~g} \mathrm{~m}^{-2}$ (Cadeddu et al., 2013). Similar steps, incoherent averaging in time then linear interpolation, are performed as with the radar to represent 
Table 4. Radar operational mode configuration settings. The radar cycles between four modes of which only the cirrus and general modes are used in this work. The modes are cycled such that the general mode is every fourth measurement and the cirrus mode is every eight at a cadence of approximately $0.5 \mathrm{~s}$ per mode.

\begin{tabular}{lrr}
\hline Radar Mode & General & Cirrus \\
\hline Average power (W) & 0.5353 & 7.146 \\
Intra-pulse period (ms) & 96 & 115 \\
Pulse width (ns) & 583 & 583 \\
Number of coded bits & 0 & 16 \\
Number of coherent averages & 5 & 6 \\
Range resolution (m) & 87.5 & 87.5 \\
\hline
\end{tabular}

MWR data onto CAPABL's grid. MWR data are a column measurement, so averaging and interpolation are only performed in time and are compared to CAPABL's column data product.

\subsubsection{Radiation}

Surface broadband radiation measurements are made at Summit by a pair of heated aspirated Kipp and Zonen CM22 pyranometers with spectral sensitivity from 0.2 to $3.6 \mu \mathrm{m}$ and a pair of aspirated Eppley Precision Infrared Radiometers (PIR) pyrgeometers, sensitive to the spectral range from 3.5 to $50 \mu \mathrm{m}$. These instruments were originally installed in August 2013 by NOAA's Global Monitoring Division. The instruments are maintained by an on-site technician at Summit, including daily removal of accumulated ice or snow. Raw data are reported as $1 \mathrm{~min}$ averages.

The pyranometers are calibrated every 2 years at NOAA's Solar Radiation Calibration Facility. The raw data are quality-controlled by NOAA's Global Monitoring Division Radiation Group. A dome correction factor for the longwave PIR is applied similar to that of Albrecht and Cox (1977). More information about the available radiation measurements at Summit is given by Miller et al. (2015).

\subsection{Direct lidar comparisons}

The first comparison performed is between CAPABL and the MPL. This is the simplest comparison to make because the data products of the MPL and CAPABL are very similar and both systems use the same operational principles. Because both instruments are lidars and have similar data streams, the results can be compared directly. As such, CAPABL's merged best-estimate voxel identifications are compared directly to the MPL's voxel identifications. Voxel identifications from CAPABL and the MPL compared for three separate time periods: July 2016, December 2016, and JulyDecember 2016. These data are given in a confusion matrix, a classification model to compare two sets of results, in Ta- ble 5, where ROIC and HOIC voxels are both combined for this comparison into "CAPABL Ice".

The time periods given in Table 5 are selected due to the solar background conditions. During the summer (e.g., July), the sun is always above the horizon at Summit. During the winter (e.g., December), the sun is always below the horizon at Summit. These two cases are highlighted to show the difference solar background makes on the data and in particular the effect on the MPL signals, which are affected strongly by solar background. CAPABL is less affected by solar background because of the receiver attenuation.

Table 5 highlights some significant sensitivity improvements of CAPABL's merged data product for daytime operations compared to the MPL. In the clear column for example, in approximately $98 \%$ of the time that CAPABL observes clear air, the MPL either agrees or lacks data to refute the CAPABL measurements over the entire study period (seen in Table 5 summing similar time periods in cells A and M). This increases to $99.5 \%$ for daylight measurements. Likewise, $96 \%$ of the data in daylight that fail the CAPABL filtering process also fail the MPL's filtering process (first line of cell P) indicating a limit of penetrable optical depth for a given power-aperture product that is a theoretical limit of all lidars. In many cases, highlighted in cells B and C in Table 5, the MPL observes clear air, while CAPABL observed clouds of some sort. This is linked directly to the Klett inversion technique requiring a strong signal derivative to highlight large backscattering ratios, approximately $>5.0$. In the case of many high clouds, the signal derivative is not strong due to noise in the perpendicular observation channel of the MPL. In comparison, the values highlighted in cells $\mathrm{E}$ and I in Table 5 are more than 2 orders of magnitude smaller because the strength of the perpendicular signal does not limit the detection range for CAPABL as it does for the MPL due to CAPABL's non-orthogonal polarization retrievals.

The sensitivity of CAPABL is linked directly to the use of non-orthogonal polarizations and analog detection. A limitation of traditional orthogonal polarization retrievals for lidar is the fact that one channel is often of higher signal strength than the other. For the MPL, the circular polarization channel is much stronger than the perpendicular polarization for low depolarization targets like clear air and liquid water. As a result, the dynamic range of the system is partially reduced by the measurement setup. For example, a depolarization of $1 \%$ would yield a difference in signal ranges of 2 orders of magnitude at a constant altitude. Therefore, for the system to observe such low depolarization, the system necessarily must sacrifice range. In terms of altitude, the lowest possible observations are set by the circular channel overlap considerations and the counting system dead time, and the highest possible observations are set by the SNR of the perpendicular signal. In contrast, CAPABL's minimum range is set by the second weakest of its four polarizations (the third channel) and maximum range is set by the second strongest of its four polarizations (the fourth channel). By design, the third 
Table 5. Confusion matrix of CAPABL and MPL processed data. The diagonal shows agreement, highlighted by bold text. The last row and last column indicate one instrument had data removed by quality control steps, also highlighted in italics. Cells B and $\mathrm{C}$ indicate enhanced sensitivity by CAPABL processing and cells $\mathrm{E}$ and I indicate enhanced sensitivity by the MPL processing. Cell P indicates both instruments lack data, implying that much of the data missed are in a regime not reachable via lidar (i.e., large optical depth). Three sets of data are given in each cell, which are identified by the last column. The first line of each cell covers the time period 1-31 July 2016. The second line of each cell covers 1-31 December 2016. The final line of each cell covers 1 July-31 December 2016.

\begin{tabular}{|c|c|c|c|c|c|c|c|c|c|}
\hline \multirow{4}{*}{ MPL clear } & \multicolumn{2}{|c|}{ CAPABL clear } & \multicolumn{2}{|c|}{ CAPABL liquid } & \multicolumn{2}{|c|}{ CAPABL ice } & \multicolumn{2}{|c|}{ CAPABL filtered } & \multirow{4}{*}{$\begin{array}{l}\text { Time period } \\
\text { July } \\
\text { December } \\
\text { All }\end{array}$} \\
\hline & (A) & $69.7 \%$ & (B) & $37.0 \%$ & (C) & $62.2 \%$ & (D) & $3.4 \%$ & \\
\hline & & $97.7 \%$ & & $64.9 \%$ & & $78.9 \%$ & & $74.5 \%$ & \\
\hline & & $83.2 \%$ & & $41.8 \%$ & & $63.9 \%$ & & $35.1 \%$ & \\
\hline \multirow[t]{3}{*}{ MPL liquid } & (E) & $0.3 \%$ & $(\mathrm{~F})$ & $56.3 \%$ & (G) & $5.5 \%$ & $(\mathrm{H})$ & $0.1 \%$ & July \\
\hline & & $0.0 \%$ & & $26.3 \%$ & & $0.2 \%$ & & $0.0 \%$ & December \\
\hline & & $0.4 \%$ & & $47.9 \%$ & & $2.0 \%$ & & $0.2 \%$ & All \\
\hline \multirow[t]{3}{*}{ MPL ice } & (I) & $0.2 \%$ & $(\mathrm{~J})$ & $3.7 \%$ & $(\mathrm{~K})$ & $29.4 \%$ & (L) & $0.5 \%$ & July \\
\hline & & $0.2 \%$ & & $8.2 \%$ & & $20.2 \%$ & & $0.3 \%$ & December \\
\hline & & $1.4 \%$ & & $8.9 \%$ & & $31.7 \%$ & & $1.1 \%$ & All \\
\hline \multirow[t]{3}{*}{ MPL filtered } & $(\mathrm{M})$ & $29.9 \%$ & $(\mathrm{~N})$ & $3.0 \%$ & $(\mathrm{O})$ & $3.0 \%$ & $(\mathrm{P})$ & $96.0 \%$ & July \\
\hline & & $2.1 \%$ & & $0.5 \%$ & & $0.6 \%$ & & $25.2 \%$ & December \\
\hline & & $15.1 \%$ & & $2.5 \%$ & & $2.4 \%$ & & $63.7 \%$ & All \\
\hline
\end{tabular}

channel is approximately half of the parallel channel's intensity and the fourth channel exceeds the perpendicular signal intensity by more than an order of magnitude, enhancing the observable range of the system in both high and low altitudes simultaneously. As a result, CAPABL is much more sensitive to a wider range of clouds and cloud properties because it is less constrained by its observable dynamic range.

The data presented in Table 5 for December observations shows a large disagreement between CAPABL and the MPL (Table 5 cell D). Here CAPABL data fail quality control filtering but MPL data are classified as clear air. The majority of the CAPABL observations filtered from the analysis are excluded because they do not meet the requirements of being a valid diattenuation observations. Either the measurements do not pass the consistency test or have an unacceptably large error. Because the diattenuation filtering is unique to CAPABL, applying this exact filtering scheme to the MPL is impossible and CAPABL data are filtered more conservatively than the MPL given the same bounds for filters common to both instruments.

The MPL and CAPABL rarely miss detecting cloud cases when they are observable by lidar. For each background condition and for the entire length of the study, not more than $3 \%$ of data are missed by one instrument when the other instrument sees cloud activity, indicated by the maximum value in cells $\mathrm{H}, \mathrm{L}, \mathrm{N}$ and $\mathrm{O}$. However, the MPL frequently mischaracterizes clouds as clear, as highlighted in cells B and C in Table 5. This is attributed as above to the signal in high background cases being hard to determine and the Klett inversion often misses thin cloud layers.

The final comparison to be made is related to the effect in this data set of multiple scattering. The effect of multi- ple scattering tends to raise the depolarization observed and delay the return of lidar signals to the system. As a direct result, the tops of thick liquid clouds in this data set can be misclassified as ice. While this effect results in a constant high depolarization bias across all measurement types for CAPABL, the overall effect is testable given the much smaller field of view of the MPL. The values in Table 5 cell $\mathrm{G}$ indicate the total measurement error caused by multiple scattering in CAPABL's data set. Over the 6-month period of study, approximately $2 \%$ of liquid voxels identified by the MPL (with smaller field of view and thus less sensitivity to multiple scattering) are misclassified by CAPABL.

\subsection{Comparisons with non-lidar data sources}

Comparisons of CAPABL data to ancillary, non-lidar, instrumentation is less straightforward than the comparison presented with the MPL. Instead of a direct comparison such as presented in Table 5, arguments about data consistency must be made. For example, within a mixed-phase cloud, both phases of water will have large size parameters (the radius of the particle, $r$ relative to the wavelength, $\lambda$, given as $2 \pi r / \lambda)$, likely greater than $50-100$ when observed by lidar, whereas at the radar wavelength the size parameter is much less than 1. In this regime, the lidar will see a scatterer well into the resonant and geometric optics regime of elastic scattering, whereas the radar will see a Rayleigh scattering target. As such, the two systems respond to different aspects of the hydrometeor population; this is one major benefit for having multiple sensors.

The expectations of multi-sensor comparisons are as follows. At $35 \mathrm{GHz}$, the MMCR signal is nominally propor- 
tional to hydrometeor size to the sixth power and is thus dominated by ice because liquid water drops are much smaller in diameter than ice in the Arctic (Turner, 2005; Shupe et al., 2006, 2013). Liquid water droplets are on the order of $10^{-5} \mathrm{~m}$, while ice crystals are on the order of $10^{-4}$ to $10^{-3} \mathrm{~m}$. By extension, the MMCR is more sensitive to liquid water droplets than clear air. One expects, therefore, to see higher radar reflectivity for ice than liquid and less still for clear air. Furthermore, as ice is much larger at Summit than liquid water droplets one expects to see higher mean Doppler velocities for ice as liquid water drops are too small to be effectively precipitated (Morrison et al., 2012). For comparison of CAPABL's data to column measurements of liquid water path with the MWR, one expects CAPABL to identify liquid overhead, while the MWR observes a positive liquid water path. Likewise, one expects to have little to no liquid water path measured for ice or clear air columns.

In light of these expectations based on observed geophysical properties, the multi-sensor comparisons are performed as follows. MWR data are processed and interpolated to the CAPABL time grid. CAPABL data are then collapsed to a column measurement based on the most radiatively important voxel type. The MWR LWP data are then assigned to one of the four possible column types: clear, ice (with HOICs labeled as HOIC or without labeled as ROIC), or liquid defined by CAPABL. The probability density function of the MWR LWP characteristics are calculated from all available data for each classification type. The cumulative distribution function is then calculated and presented in Fig. 6. The data that have been filtered by lidar are removed. Raw radar data that have been interpolated to CAPABL's data grid are assigned using CAPABL's data identifiers. The five data types (clear air, cloud liquid, ROIC, HOIC, and filtered) are each distinctly binned together. The probability density function of the radar characteristics and the cumulative distribution function are also calculated from all available data. The data that have been filtered by lidar are removed. The cumulative distribution functions of the remaining four variables are shown in Fig. 6 for the first two radar Doppler moments and its SNR. In this time period, CAPABL has data available for $75.3 \%$ of the total voxels where there is filtered MMCR data available. Note that though they contain and represent the same data, this work will choose to represent instrument comparisons in terms of their cumulative distribution functions as opposed to the probability density function. Both facilitate comparisons of large quantities of data but cumulative distribution functions allow simple comparisons of differences of shape and median, whereas the probability density function allows for investigations of modes and biases.

It can be seen in Fig. 6 that the expected relationships between the lidar, MWR, and MMCR hold very well. Nearly $69 \%$ of all columns tagged as containing liquid by CAPABL have non-zero LWP (here zero and non-zero are taken below and above the error bounds of the measurement, respectively). Almost $91 \%$ of columns tagged as ROIC, $90 \%$

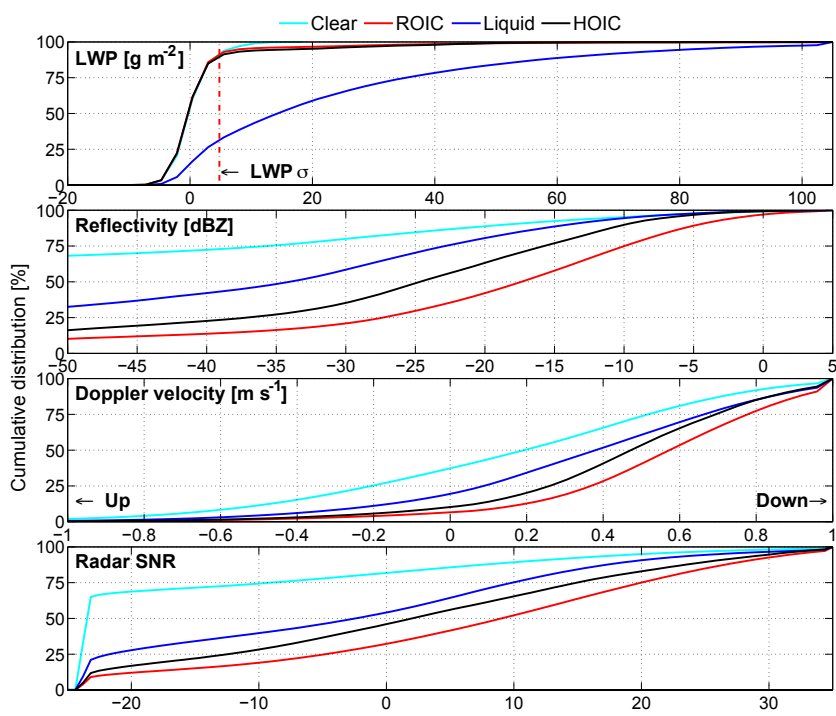

Figure 6. Cumulative distribution functions of co-located ICECAPS data parsed by CAPABL classification type. All data from July 2016 to December 2016, approximately 54 million radar voxels for each Doppler moment and 148000 MWR column measurements, are collected and identified. Note that the average LWP uncertainty is given for the entire study period and that here a positive mean Doppler velocity is defined towards the zenith-pointing radar system or downwards. For the LWP uncertainty, assuming an effective radius of $r_{\mathrm{e}}=10 \mu \mathrm{m}$, a density of water of $\rho=1000 \mathrm{~kg} \mathrm{~m}^{-3}$, and using the approximate relation LWP $=2 \tau r_{\mathrm{e}} \rho / 3$ (Bendix, 2002) yields a threshold for optical depth of $\tau=0.75$.

tagged with HOIC, and $91 \%$ tagged as clear do not have LWPs above the error bounds of the MWR measurement. CAPABL can mis-identify very low cloud and precipitation, below approximately $200 \mathrm{~m}$, as clear air columns because there is no identifiable cloud voxels in the instrument's valid sample volume. In terms of comparison to radar, this is not a problem as no mask is returned and is thus not considered, but in terms of column measurements this will yield an error in identification. More strenuous filtering of the column mask by the flag indicating obscuration described in Sect. 3.3 increase the percent of clear air, ROIC, and HOIC data with zero LWP on the order of approximately $5 \%$. From the MWR perspective, $83 \%$ of instances where non-zero LWP are observed are either marked as liquid or obscured by CAPABL. Clear air, ROIC, and HOIC comprise the other 3,10 , and $4 \%$, respectively. It should be noted that scattering of surface radiation has been shown to cause erroneous nonzero liquid water path of thick ice layers (Pettersen et al., 2016). More strenuous filtering of the column mask by the flag indicating obscuration similarly lowers these percentages to reduce the overall clear air, ROIC, and HOIC instances with non-zero LWP.

CAPABL has valid observations at $75 \%$ of locations where valid MMCR data are observed over the time period of interest. The reflectivity of clear air voxels is much lower 
than that of ice and liquid water. More than $89 \%$ of all voxels identified by CAPABL as clear fall below $-20 \mathrm{dBZ}$, whereas only $42 \%$ identified as ice fall below the same threshold. This is confirmed with radar SNR where $69 \%$ of all clear air data fall below the SNR threshold of $-20 \mathrm{dBZ}$ (this value is $72 \%$ for the threshold of $-14 \mathrm{dBZ}$ used by Shupe et al., 2013). Similarly, the largest scatterers, ROICs and HOICs, have higher SNR. Note that HOICs have a lower median reflectivity than ROICs in Fig. 6. This is not true in the radar cirrus mode above $3 \mathrm{~km}$ (cirrus mode is more sensitive than the general mode designed for higher-altitude observations; Clothiaux et al., 1999). The cumulative distributions for the radar cirrus mode (not shown) have reflectivity values for ROICs and HOICs that nearly overlap. This change in reflectivity and inconsistency between radar modes could indicate two things: first that HOICs are possibly occurring in thinner more tenuous clouds on average than ROICs with smaller ice particles, or second that ground-based lidar measurements have a sampling bias that only allows observations of HOICs in thinner clouds.

ROIC has the highest Doppler velocity, with HOIC and liquid falling slower. ROIC has a median mean Doppler velocity of approximately $0.57 \mathrm{~m} \mathrm{~s}^{-1}$ downward, while HOIC and liquid are 0.47 and $0.38 \mathrm{~m} \mathrm{~s}^{-1}$, respectively, both in the downward direction. The occurrence of falling liquid indicates mixed-phase voxels where CAPABL is more sensitive to the liquid phase and MMCR to the ice phase. The slight skewness of the clear air identifier to downward mean Doppler velocity, indicated by the non-zero median, indicates that some ice is being tagged as clear air by CAPABL, which is known to occur at the very top of clouds and below very optically thick clouds due to the Klett inversion, and is especially prominent as mentioned with the MWR results where low (below approximately 100-200 m) thick clouds are observed. The reduced Doppler velocity of HOIC is anticipated due to the enhanced aerodynamic drag associated with their orientation (Westbrook et al., 2010). This is a clear verification that HOIC identification by CAPABL based on the novel diattenuation technique of Neely et al. (2013) is physically consistent.

\section{Discussion and conclusion}

\subsection{Applicability to other lidar sensors}

Polarimetric lidar systems are widely deployed. Nott and Duck (2011) and references therein lists many other groundbased lidar deployment sites in the polar regions such as Syowa, Antarctica; South Pole, Antarctica; Eureka, Canada; and Barrow, Alaska. Further, the CALIOP lidar on board the CALIPSO satellite uses analog detection, and regularly observes the polar regions (Winker et al., 2009). Due to varying configurations and approaches by other lidars, it is difficult to specifically identify how well other comparable systems represent cloud properties, but the unique instrument suite at Summit and the novel lidar configuration of the CAPABL system enable such an analysis.

Lidar systems are fundamentally limited by their receiver dynamic range. For polarized systems, like CAPABL and the MPL, observational range is inversely related to atmospheric depolarization. Assuming a limited dynamic range of 5 orders of magnitude, this can be parsed either for range, arising from the $A / R^{2}$ term in the lidar equation, or by depolarization. For depolarization ratios of $1 \%$, this leaves only 3 orders of magnitude for changes in range. The altitude range is limited on top by weak perpendicular signals and on the bottom by strong parallel signals. Fundamentally, this limits the effective observational range that has the effect of biasing attribution of cloud properties on, for example, evaluating the radiation budget. At Summit, CAPABL provides a fully merged data product that covered $34 \%$ of the column from 0 to $8 \mathrm{~km}$ for July to December 2016 . Using only orthogonal components from analog and photon counting results in only $25 \%$ coverage. In comparison to CAPABL, the MPL observed $19 \%$ of the column above Summit in summer (CAPABL observes $25 \%$ for the fully merged mask and only $18 \%$ for the orthogonal components) and $44 \%$ in winter (CAPABL observes $45 \%$ for the fully merged mask and only $31 \%$ for the orthogonal components). The data are split again, noting that CAPABL is more conservatively filtered in the winter based on its diattenuation filtering. Thus, the general impact of lidar observations is site and lidar specific (as is analyzed for Summit in Sect. 6.2) but should be recognized as a possible cause for interpretive bias.

Potential shortcomings of limited counting system dynamic range are clearly visible in the data shown in this work. Figure 5 shows the FO of clouds above Summit using analog detection and photon counting detection, as well as orthogonal and non-orthogonal polarization retrievals. Each of these observational methods can handle slightly different altitude ranges based on the signal strength and system sensitivity to those signals. The results indicate that the occurrence of liquid water can be underestimated by as much as $30 \%$ depending on the counting type. This limitation is due to low-level clouds causing saturation in photon counting detection, especially in the stronger polarization channels, which overestimates depolarization, and consequently the depolarization ratio, which makes liquid clouds look like ice clouds. Photon counting systems, such as the polarization sensitive MPL, are susceptible to this sort of underestimation of liquid water clouds. In the opposite direction, analog detection underestimates total cloud FO, on the order of 4 to $22 \%$, because it is insensitive to higher, optically thinner ice clouds that are clearly visible using photon counting detection. In either case, the choice of counting system type, or indeed receiver polarization selection, limits the altitude range of interest and by extension the clouds to be observed. The unique configuration of CAPABL allows for these assessments to be made and optimized. 
Another clear limitation of lidar sensors is their inability to observe the entire vertical column in the presence of optically thick clouds with visible optical depths on the order of 3 or greater. This limitation is clearly visible in CAPABL's data and in particular its incomplete coverage of the entire altitude range above Summit during times of mixedphase clouds. Similar limitations are to be expected from both ground-based systems and space-based systems.

\subsection{Impact on attribution of cloud effects on the surface radiation budget}

In a similar method to data comparisons with LWP, comparisons of CAPABL data to observed downwelling and upwelling longwave (LW) and shortwave (SW) radiation fluxes have been performed. These comparisons elucidate the drawbacks of certain lidar methodologies and optimizes CAPABL's approach to provide a best estimate. The cumulative distribution functions of downwelling and upwelling LW and $\mathrm{SW}$ radiation measurements as well as the net radiation, defined as net $=\mathrm{LW} \downarrow+\mathrm{SW} \downarrow-\mathrm{LW} \uparrow-\mathrm{SW} \uparrow$, are given in Fig. 7 for CAPABL's merged best-estimate data product, parsed into column types: clear air, ice (with and without HOIC), and liquid bearing. Figure 7 shows some simple relationships that are examined for consistency with previous studies. The median value of downwelling LW radiation is higher for liquid clouds than it is for ice clouds, which is higher still than for clear air. This is expected based on many previous results including those of Curry et al. (1996), Shupe and Intrieri (2004), and Miller et al. (2015). Likewise, the downwelling SW flux is highest for clear air and reduced for ice clouds, which is further reduced for liquid clouds. This shows the dominance of cloud visible optical depth by liquid clouds, which is well described by Shupe and Intrieri (2004), Stevens and Bony (2013), and Miller et al. (2015). The upwelling LW measurements are highest for liquid cloud scenes, which can be understood based on the enhanced downwelling LW radiation and emission that scales with surface temperature to the fourth power. Miller et al. (2017) showed warmer surface temperatures occur with liquid clouds overhead. Finally, upwelling SW is simply the scaled version of the downwelling SW, scaled by surface albedo. These results are all expected and provide further validation that the CAPABL cloud identification procedure is acting as expected.

The median values of all distributions for the three CAPABL classification types, analog, photon counting, and merged, and all four radiation types and the net radiation are listed in Table 6. The merged column is our best estimate through signal combinations so that a difference between merged and analog or merged and photon counting indicates limitations for those stand-alone techniques. For example, the percent difference for the downwelling longwave radiation for clear air and ROIC is on the order of 5-10\% (seen in Table 6 in the "Downwelling longwave" section).

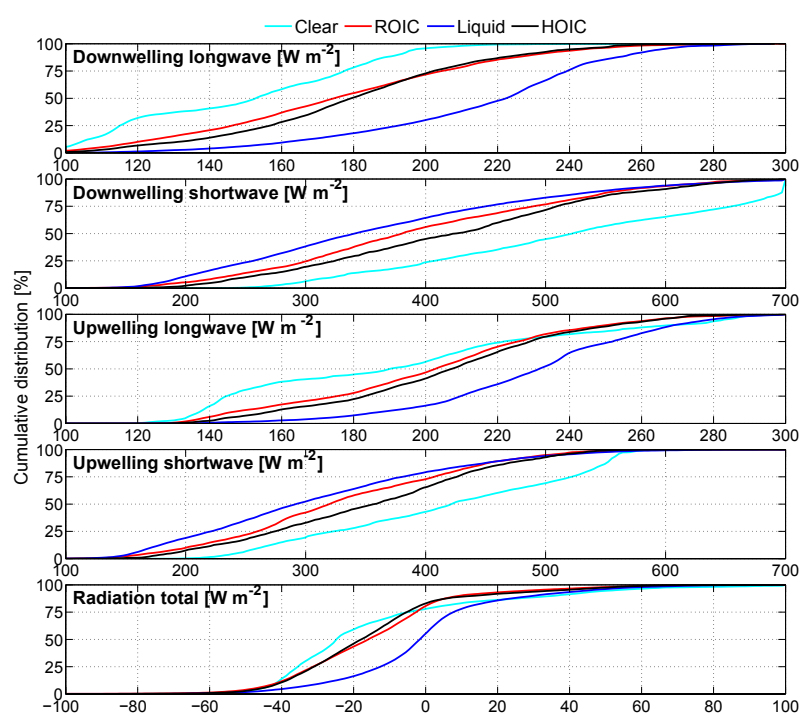

Figure 7. Cumulative distribution function of downwelling and upwelling radiation data at the surface parsed by CAPABL column classification type.

This difference for analog is attributed to difficulty measuring the whole column of ice especially in the polar summer with just orthogonal polarization retrievals. Due to its lower designed sensitivity, the analog clear air classification misses some ice clouds that contaminate the clear air classification. The difference for photon counting is attributed to saturation. The ROIC classification is contaminated by low liquid clouds artificially raising the overall downwelling longwave effect. The same affects the downwelling shortwave measurements on the order of $10 \%$ (seen in Table 6 in the "Downwelling shortwave" section). For photon counting measurements, contamination from liquid clouds lowers the downwelling shortwave component. For the total radiative components, the lack of sensitivity of the analog channel artificially raises the clear air radiative balance towards the values for ice clouds. For the photon counting component, saturation raises the ROIC radiative balance towards the values for liquid clouds. In all cases, traditional lidar data used to attribute radiative fluxes will introduce large uncertainties based on the lidar's inability to measure the whole atmospheric column related to its limited dynamic range. Enhancing measurements as done in this work with non-orthogonal polarization retrievals as well as analog and photon counting detection allows for a more complete attribution of radiative effects linked to cloud properties.

A second method of analyzing the radiative importance of this work is to use literature values to estimate cloud radiative effects. Figure 5 gives the FO of voxel types in the column above CAPABL. Using this FO, literature values such as those presented by Miller et al. (2015) can be used to estimate misattribution of cloud radiative effects. Figure 5 shows 
Table 6. Median values of the probability distribution function for each data processing type for each radiation component. Each radiation component is measured at the surface in units of $\mathrm{W} \mathrm{m}^{-2}$. Net flux is calculated using the relation: total $=\mathrm{LW} \downarrow+\mathrm{SW} \downarrow-\mathrm{LW} \uparrow-\mathrm{SW} \uparrow$.

\begin{tabular}{|c|c|c|c|c|c|}
\hline Type & Merged & Analog & $\mathrm{PC}$ & $\begin{array}{r}\% \text { difference } \\
\text { merged and analog }\end{array}$ & $\begin{array}{r}\% \text { difference } \\
\text { merged and PC }\end{array}$ \\
\hline \multicolumn{6}{|c|}{ Downwelling longwave } \\
\hline Clear & 153.3 & 162.1 & 155.3 & 5.6 & 1.2 \\
\hline ROIC & 175.1 & 181.2 & 194.3 & 3.4 & 10.4 \\
\hline Liquid & 222.9 & 224.1 & 213.5 & 0.5 & 4.3 \\
\hline HOIC & 179.8 & 179.2 & 179.9 & 0.4 & 0.0 \\
\hline \multicolumn{6}{|c|}{ Upwelling longwave } \\
\hline Clear & 190.6 & 209.8 & 204.3 & 9.6 & 6.9 \\
\hline ROIC & 203.2 & 206.0 & 214.3 & 1.4 & 5.3 \\
\hline Liquid & 232.0 & 232.9 & 227.6 & 0.4 & 2.0 \\
\hline HOIC & 207.8 & 206.4 & 207.5 & 0.6 & 0.1 \\
\hline \multicolumn{6}{|c|}{ Downwelling shortwave } \\
\hline Clear & 524.1 & 571.7 & 539.8 & 8.7 & 3.0 \\
\hline ROIC & 381.8 & 356.6 & 342.8 & 6.8 & 10.8 \\
\hline Liquid & 342.5 & 342.4 & 379.2 & 0.0 & 10.2 \\
\hline HOIC & 426.2 & 418.9 & 418.0 & 1.7 & 1.9 \\
\hline \multicolumn{6}{|c|}{ Upwelling shortwave } \\
\hline Clear & 422.9 & 462.6 & 426.6 & 9.0 & 0.9 \\
\hline ROIC & 321.2 & 309.4 & 294.6 & 3.7 & 8.6 \\
\hline Liquid & 293.3 & 293.0 & 323.8 & 0.1 & 9.9 \\
\hline HOIC & 359.2 & 352.4 & 353.2 & 1.9 & 1.7 \\
\hline \multicolumn{6}{|l|}{ Total } \\
\hline Clear & -24.3 & -23.7 & -25.0 & -2.4 & -2.7 \\
\hline ROIC & -15.5 & -14.7 & -9.0 & -4.9 & -53.3 \\
\hline Liquid & -1.1 & -0.7 & -4.0 & -48.2 & -112.2 \\
\hline HOIC & -17.4 & -17.2 & -16.1 & -1.1 & -7.4 \\
\hline
\end{tabular}

a difference of approximately $30 \%$ from analog to photon counting for liquid FO. This difference can be used to approximate an error in cloud radiative forcing using the results from Fig. 7 from Miller et al. (2015). Using an average difference of $30 \%$, this time period of fractional occurrence of liquid clouds equates to an error in longwave cloud radiative effect of approximately $10 \mathrm{~W} \mathrm{~m}^{-2}$. Miller et al. (2015) find an average of $33 \mathrm{~W} \mathrm{~m}^{-2}$ for cloud radiative forcing at Summit, suggesting that using conventional lidar approaches to infer radiative impacts could under-represent forcing by as much as one-third. The CAPABL approach improves the situation significantly, leading to better attribution of cloud effects on radiative fluxes.

\subsection{Main conclusions}

This work has demonstrated three key points. The first point is that cloud-phase classification by polarimetric lidar is sensitive not only to the cloud phase but also to lidar design properties such as receiver polarization, detection schemes, and backscattered signal count rate and, by extension, cloud macrophysical properties such as base height (or range) and optical depth. The second point is the utility of nonorthogonal polarization measurements to improve cloud classifications. By employing multiple planes of polarization in the lidar receiver, in the case of CAPABL four linear planes, the diversity in backscattered intensity may be handled more judiciously, making the characterization of cloud types more accountable. This effectively spreads the required dynamic range of signals among the multiple polarization measurements. By measuring additional planes of polarization beyond what is required for geophysical retrievals allows the CAPABL system to self-analyze limitations in a channel's performance, correct some of the behavior through nonorthogonal signal combinations, and optimize the use of the different channels for different cloud scattering conditions. In high-dynamic-range targets, like optically thick liquidonly or mixed-phase clouds, systematic errors can cause a misrepresentation in traditional polarization-sensitive lidars 
of liquid clouds as ice clouds. Here this is shown to occur on the order of $30 \%$ of the time for CAPABL but is correctable using the presented novel polarization scheme. Finally, this work has analyzed the effects of lidar data in terms of radiative attribution. Using a particular detection system such as photon counting, orthogonal polarization measurements can dramatically mis-represent cloud radiative effect. Using radiation measurements from Summit, errors in attribution of radiative scenes related to cloud phase can be on the order of $10 \%$ of the net radiation. Using cloud fraction as an estimator with previously published radiative estimates of Miller et al. (2015) suggests an even higher $30 \%$ misattribution.
Code and data availability. All data collected by the ICECAPS program are publicly available at the NSF Arctic Data Center at https://arcticdata.io/catalog/\#view/urn:uuid: f783f072-e5e0-454c-9c9d-136935e1cb7c (Walden et al., 2010). Radiation data collected by the NOAA are publicly available at ftp://aftp.cmdl.noaa.gov/data/radiation/baseline/sum/. The code developed to process the CAPABL data is available by request from the authors. 
Appendix A: Derivation of generalized depolarization and diattenuation expressions

From the general form of the Stokes vector lidar equation given in Eq. (1), the number of photons to be observed in any arbitrary linear polarization channel can be derived. Assuming that CAPABL (1) emits a linear polarized signal at angle $\phi$, yielding the simplification

$\mathbf{M}_{T_{x}}\left(\boldsymbol{k}_{\mathrm{i}}\right) \boldsymbol{S}_{T_{x}}=\left[\begin{array}{llll}1 & \cos (2 \phi) & \sin (2 \phi) & 0\end{array}\right]^{T}$,

(2) only measures linear polarized signal at angle $\theta$ from the reference transmit polarization (Neely et al., 2013, Eq. 15 with $\left.A\left(\Gamma_{\mathrm{wp}}\right)=\mathbf{M}_{R_{x}}(2 \theta)\right)$ yielding the simplification

$\mathbf{M}_{R_{x}}\left(\boldsymbol{k}_{\mathrm{s}}\right)=\frac{1}{2}\left[\begin{array}{cccc}1 & \cos (2 \theta) & \sin (2 \theta) & 0 \\ 1 & \cos (2 \theta) & \sin (2 \theta) & 0 \\ 0 & 0 & 0 & 0 \\ 0 & 0 & 0 & 0\end{array}\right]$

and (3) using the definition of the backscattering phase matrix (Hayman and Thayer, 2012; Neely et al., 2013)

$$
\begin{aligned}
& \mathbf{F}\left(\boldsymbol{k}_{\mathrm{i}},-\boldsymbol{k}_{\mathrm{i}}, R\right)= \\
& {\left[\begin{array}{cccc}
F_{11}(R) & F_{12}(R) & 0 & 0 \\
F_{12}(R) & F_{22}(R) & 0 & 0 \\
0 & 0 & F_{33}(R) & F_{34}(R) \\
0 & 0 & F_{34}(R) & F_{44}(R)
\end{array}\right],}
\end{aligned}
$$

the number of photons to be observed in any arbitrary linear polarization channel is given in Eq. (A4) as

$$
\begin{aligned}
& N_{M}(R)=\xi(R)\left[F_{11}(R)+\cos (2 \theta) F_{12}(R)+\cos (2 \phi)\right. \\
& \left.\left(F_{12}(R)+\cos (2 \theta) F_{22}(R)\right)+\sin (2 \theta) \sin (2 \phi) F_{33}(R)\right] .
\end{aligned}
$$

Here, all constant terms of Eq. (1), which will cancel when taking signal ratios, are lumped into the term $\xi(R)$ such as the measurement solid angle, geometric overlap, range resolution, and atmospheric transmission.

The number of measured photons incident upon the photodetector, $N_{M}(R)$, is a function of transmitted and received polarization angle $\phi$ and $\theta$, respectively, and is related to the scattering phase matrix terms, $F_{11}(R), F_{12}(R), F_{22}(R)$, and $F_{33}(R)$, which are all functions of range. For CAPABL, $\phi=45^{\circ}$; applying this constraint to Eq. (A4) cancels the functional dependency on $F_{22}(R)$ by design. Thus, using three distinct receiver polarization channels $-\theta_{1}, \theta_{2}$, and $\theta_{3}$ - one can create a set of three simultaneous equations which can be inverted to calculate the Mueller matrix terms of interest that describe backscattering coefficient $\left(F_{11}\right)$, volume depolarization $\left(F_{33} / F_{11}\right)$, and volume diattenuation $\left(F_{12} / F_{11}\right)$.
This set of equations is given in Eq. (A5) as

$$
\begin{aligned}
& {\left[\begin{array}{l}
N_{1}(R) \\
N_{2}(R) \\
N_{3}(R)
\end{array}\right]=} \\
& \xi(R)\left[\begin{array}{lll}
1 & \cos \left(2 \theta_{1}\right) & \sin \left(2 \theta_{1}\right) \\
1 & \cos \left(2 \theta_{2}\right) & \sin \left(2 \theta_{2}\right) \\
1 & \cos \left(2 \theta_{3}\right) & \sin \left(2 \theta_{3}\right)
\end{array}\right]\left[\begin{array}{l}
F_{11}(R) \\
F_{12}(R) \\
F_{33}(R)
\end{array}\right] \\
& \rightarrow \boldsymbol{N}=\mathbf{A} F .
\end{aligned}
$$

The general matrix inverse of $\mathbf{A}$ is given in Eq. (A6) as

$\mathbf{A}^{-1}=$

$\frac{1}{\zeta}\left[\begin{array}{c}\sin \left(2 \theta_{2}-2 \theta_{3}\right) \\ \sin \left(2 \theta_{3}\right)-\sin \left(2 \theta_{2}\right) \\ \cos \left(2 \theta_{2}\right)-\cos \left(2 \theta_{3}\right)\end{array}\right.$

$$
\begin{gathered}
\sin \left(2 \theta_{3}-2 \theta_{1}\right) \\
\sin \left(2 \theta_{1}\right)-\sin \left(2 \theta_{3}\right) \\
\cos \left(2 \theta_{3}\right)-\cos \left(2 \theta_{1}\right)
\end{gathered}
$$$$
\left.\begin{array}{c}
\sin \left(2 \theta_{1}-2 \theta_{2}\right) \\
\sin \left(2 \theta_{2}\right)-\sin \left(2 \theta_{1}\right) \\
\cos \left(2 \theta_{1}\right)-\cos \left(2 \theta_{2}\right)
\end{array}\right] \text {. }
$$

Note that the matrix $\mathbf{A}$ and the matrix inverse $\mathbf{A}^{-1}$ are not functions of range but only of the selected receiver polarizations. The term

$$
\begin{gathered}
\zeta=\cos \left(2 \theta_{3}\right)\left(\sin \left(2 \theta_{2}\right)-\sin \left(2 \theta_{1}\right)\right)+\cos \left(2 \theta_{1}\right)\left(\sin \left(2 \theta_{3}\right)\right. \\
\left.-\sin \left(2 \theta_{2}\right)\right)+\cos \left(2 \theta_{2}\right)\left(\sin \left(2 \theta_{1}\right)-\sin \left(2 \theta_{3}\right)\right)
\end{gathered}
$$

is introduced in Eq. (A6) as a constraint on the validity of the inversion where $\zeta=0$ results in a degenerate inversion because of receiver polarization selection. This happens for example when two angles are equal or $180^{\circ}$ separated.

Volume depolarization,

$d\left(R, \theta_{i}\right)-1=\frac{F_{33}\left(R, \theta_{i}\right)}{F_{11}\left(R, \theta_{i}\right)}=$

$\frac{\left(\cos \left(2 \theta_{3}\right)-\cos \left(2 \theta_{2}\right)\right) N_{1}(R)+\left(\cos \left(2 \theta_{1}\right)-\cos \left(2 \theta_{3}\right)\right) N_{2}(R)+\left(\cos \left(2 \theta_{2}\right)-\cos \left(2 \theta_{1}\right)\right) N_{3}(R)}{\sin \left(2 \theta_{2}-2 \theta_{3}\right) N_{1}(R)+\sin \left(2 \theta_{3}-2 \theta_{1}\right) N_{2}(R)+\sin \left(2 \theta_{1}-2 \theta_{2}\right) N_{3}(R)}$,

and volume diattenuation,

$D\left(R, \theta_{i}\right)=\frac{F_{12}\left(R, \theta_{i}\right)}{F_{11}\left(R, \theta_{i}\right)}=$

$\frac{\left(\sin \left(2 \theta_{3}\right)-\sin \left(2 \theta_{2}\right)\right) N_{1}(R)+\left(\sin \left(2 \theta_{1}\right)-\sin \left(2 \theta_{3}\right)\right) N_{2}(R)+\left(\sin \left(2 \theta_{2}\right)-\sin \left(2 \theta_{1}\right)\right) N_{3}(R)}{\sin \left(2 \theta_{2}-2 \theta_{3}\right) N_{1}(R)+\sin \left(2 \theta_{3}-2 \theta_{1}\right) N_{2}(R)+\sin \left(2 \theta_{1}-2 \theta_{2}\right) N_{3}(R)}$,

can be expressed in terms of arbitrary observation angles assuming the condition $\zeta \neq 0$ (for CAPABL $\zeta \approx-2$ calculated from receiver polarizations via atmospheric calibration performed for each measurement).

\section{Appendix B: CAPABL's nonlinear photon counting}

CAPABL's photon counting system is subject to pulse pileup, as is the case with most photon counting systems. This pileup results in detector pulses occurring too close in time for the counting system to uniquely identify individual pulses, resulting in systematic underrepresentation of photon count rate. The models introduced to correct this problem are based on the work of Donovan et al. (1993), Whiteman (2003), 
and Liu et al. (2009) using a calibration data set taken during a clean air period at Summit in May 2015. The neutraldensity filter was removed from the receiver optical path on a clear air day to increase the observed count rate and also extend the vertical range of calibration data. Data were concatenated based on the work of Newsom et al. (2009), with the main difference being that profiles were backgroundsubtracted before analysis (note that this is the only case in this manuscript where such concatenation is performed). From these data, the analog profile is taken as the ideal count rate. These data are plotted in Fig. B1 with two correction methods fit to the data using a Levenberg-Marquardt nonlinear least squares solver. These saturation models are given as

$S_{\mathrm{obs}}=\frac{S_{0}}{1+\tau_{\mathrm{NP}} S_{0}}$

and

$S_{\text {obs }}=S_{0} \exp \left(\tau_{\mathrm{P}} S_{0}\right)$,

referred to as non-paralyzable and paralyzable, respectively. The fit parameter for non-paralyzable is the dead time $\tau_{\mathrm{NP}}$ and for paralyzable $\tau_{\mathrm{P}}$.

To convert from the observed photon count number to observed photon count rate, the simple linear transformation

$N_{\mathrm{obs}}=S_{\mathrm{obs}} \times S_{\mathrm{PP}} \times T_{\mathrm{PB}}$

is used where $N_{\text {obs }}$ is the observed photon count number per bin, $S_{\mathrm{obs}}$ is the observed photon count rate per shot, $S_{\mathrm{PP}}$ is the number of laser shots integrated per profile, and $T_{\mathrm{PB}}$ is the two way travel time of light per range bin.

Inserting Eq. (B3) into Eq. (B1) and performing a propagation of error analysis, based on Taylor series expansion for standard error propagation assuming no data covariance, yields the shot noise error for the corrected photon count number per bin given as

$\sigma_{N}=S_{\mathrm{PP}} T_{\mathrm{PB}} \sqrt{\frac{N_{\mathrm{obs}}^{4} \sigma_{\tau_{\mathrm{NP}}}^{2}+S_{\mathrm{PP}}^{2} T_{\mathrm{PB}}^{2} \sigma_{N_{\mathrm{obs}}}^{2}}{\left(S_{\mathrm{PP}} T_{\mathrm{PB}}-\tau_{\mathrm{NP}} N_{\mathrm{obs}}\right)^{4}}}$.

Equation (B4) indicates that the error in corrected photon count rate is a function of the count error $\sigma_{N_{\mathrm{obs}}}$, which conform to Poisson statistics, and the error in the model fit parameter $\tau_{\mathrm{NP}}$. This error is estimated during the fitting procedure using the fit confidence bounds. Note that if, and only if, $\tau_{N P}$ is exactly zero (i.e., $\tau_{\mathrm{NP}}=0$ and $\sigma_{\tau_{\mathrm{NP}}}=0$ ), the counting error will be simply $\sigma_{N_{\mathrm{obs}}}$.

The calibration data used for this analysis are presented in Fig. B1. As each measurement is subject to some measurement error, Poisson counting error for photon counting and electrical noise for the analog detection, this fit was calculated using the SNR as a data weight such that higher SNR data are given higher weights. The results of this weighted analysis indicate that the dead time is approximately $0.1 \mathrm{~ns}$ higher than the unweighted analysis which ignores measurement errors in the fit.

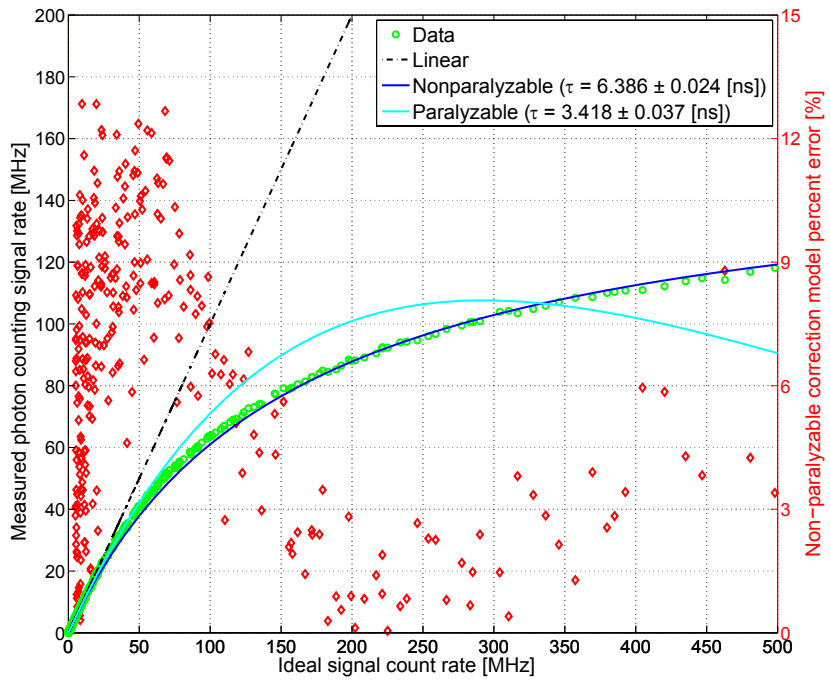

Figure B1. Saturation analysis of the CAPABL photon counting channel using the theory developed by Donovan et al. (1993), Whiteman (2003), and Liu et al. (2009). The ideal signal count rate is found by normalizing the analog detection channel to the photon counting channel in a region where both are acting linearly which is about $1 \mathrm{MHz}$ count rate. The measured count rate is then taken directly from photon counting measurements. The paralyzable and non-paralyzable models are then fit using a Levenberg-Marquardt weighted nonlinear least squares fitting algorithm of the observed calibration data. The $1 \sigma$ confidence bound is given for each dead time fit parameter. Finally, the percent error of the correction model is given relative to the ideal count rate on the right ordinate as diamonds.

\section{Appendix C: Interpretation of liquid, ice, and clear air voxels from CAPABL's first 4 months}

To add to the analysis presented in Fig. 3, a complete boxand-whisker plot for liquid, ice, and clear air is given in Fig. C1. Note here that randomly oriented ice crystals and horizontally oriented ice crystals are both included as ice. Figure $\mathrm{C} 1$ indicates 3 prominent features. First, as mentioned in the main text, the median altitude of liquid voxels is not constant between analog, photon counting, and saturationcorrected photon counting (SCPC) for either orthogonal or non-orthogonal retrievals. The second feature is seen in the clear sky data where there is increased sensitivity of the photon counting channel over the analog channel and increased sensitivity of the non-orthogonal polarization retrievals over the orthogonal versions. This increased sensitivity is seen by the increase in whisker range of approximately $1 \mathrm{~km}(0.96$, $0.70,0.34$, and $0.55 \mathrm{~km}$ for July, August, September, and October for saturation-corrected photon counting and ana$\log$ to the 95th percentile, respectively, or $1.17,1.12,0.99$, and $0.83 \mathrm{~km}$ to the inner fence), indicating the presence of more high-altitude clear air voxels that pass the quality control standards specified in Table 2. As a result of the increased sensitivity, the median altitude of the clear-sky data 
shifts upwards as well $(0.29,0.29,0.36$, and $0.31 \mathrm{~km}$ for July, August, September, and October for SCPC, respectively). The final feature is the relative consistency of the occurrence of ice for all methods. The median altitude of the iceidentified data shifts slightly upwards again due to increased sensitivity between analog and photon counting $(0.05,0.23$, 0.36 , and $0.23 \mathrm{~km}$ for July, August, September, and October for saturation-corrected photon counting and analog, respectively) but the boxes cover similar altitude ranges, especially for July. Comparing the whiskers for the non-orthogonal and orthogonal polarization retrievals within a month indicates that the increased sensitivity gained by using non-orthogonal polarization retrievals does not change the geophysical interpretation of the ice-identified data when saturation is of little concern (shifts of $0.26,0.08,0.21$, and $0.10 \mathrm{~km}$ for July, August, September, and October for analog to the 95th percentile, respectively, or $0.18,0.13,0.21$, and $0.18 \mathrm{~km}$ to the inner fence are observed), i.e., when signals are of similar strength or when signal rates are less than or on the order of approximately $1 \mathrm{MHz}$.
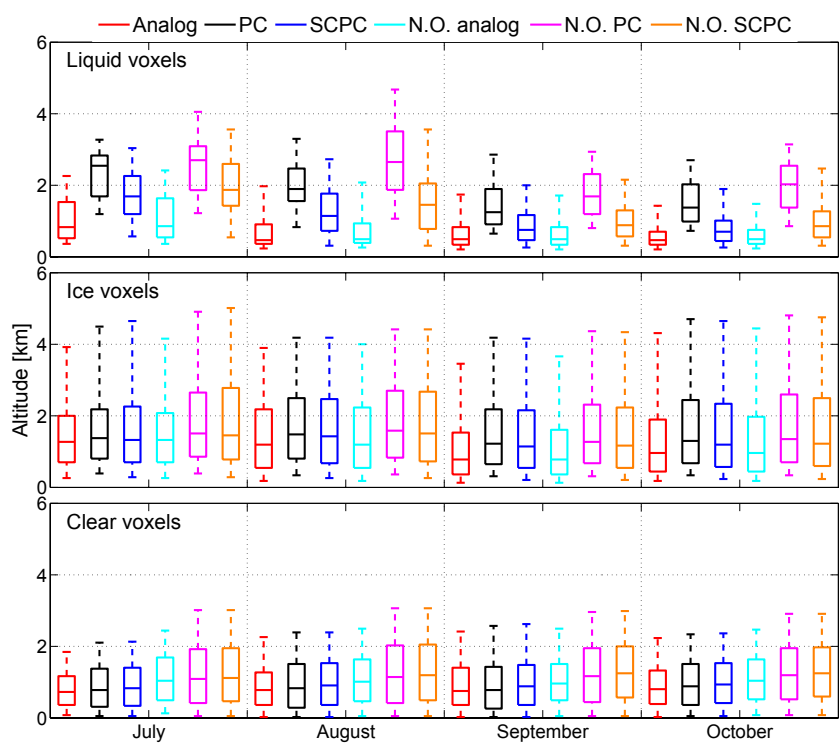

Figure C1. CAPABL data from July 2015 to October 2015 binned into liquid, ice, or clear air. The median is indicated by a line through the box, the 25 th to 75 th percentile ranges complete the box, and the whiskers extend to the 5th and 95th percentiles. PC, SCPC, and N.O. stand for photon counting, saturation-corrected photon counting, and non-orthogonal, respectively. The channel sensitivity can be seen looking at the clear voxels where analog is expected to be less sensitive than PC and orthogonal less sensitive than non-orthogonal. 
Author contributions. RAS developed the processing code for CAPABL and MPL data, the data merging procedure, and performed the multi-sensor comparison and radiation analysis. MDS provided MMCR data. DDT provided the MWR and MPL raw data and performed the MWR optimal estimation retrievals. JPT and RRN served as advisors for RAS for CAPABL specific technical tasks and RRN, MDS, and DDT contributed scientific context. The ICECAPS instrument is maintained by technicians from Polar Field services in close coordination with RAS, RRN, MDS, and DDT. RS prepared the manuscript with contributions from RRN, JPT, MDS, and DDT.

Competing interests. The authors declare that they have no conflict of interest.

Acknowledgements. This material is based upon work supported by the National Science Foundation Graduate Research Fellowship Program under grant DGE 1144083 and National Science Foundation grants PLR-1303864, PLR-1303879, PLR-1314156, PLR-1304692, and ATM-0454999. The National Center for Atmospheric Research is sponsored by the National Science Foundation. Ryan R. Neely is funded by the National Centre for Atmospheric Science. The authors would like to thank the staff and science technicians at Summit, especially Hannah James and Samuel Dorsi, as well as Polar Field Services for their support and dedication to help collect data and maintain instrumentation. Additionally, the authors would like to thank Claire Pettersen for helpful discussions pertaining to microwave radiometer retrievals. The authors would also like to thank David Longenecker for providing radiation data. ICECAPS MPL data were provided by the US Department of Energy Atmospheric Radiation Measurement Program while MMCR data were provided by the NOAA Earth System Research Laboratory.

Edited by: Manfred Wendisch

Reviewed by: three anonymous referees

\section{References}

Albrecht, B. and Cox, S. K.: Procedures for Improving Pyrgeometer Performance, J. Appl. Meteorol., 16, 188-197, https://doi.org/10.1175/15200450(1977)016<0190:PFIPP>2.0.CO;2, 1977.

Alvarez, J. M., Vaughan, M. A., Hostetler, C. A., Hunt, W. H., and Winker, D. M.: Calibration Technique for PolarizationSensitive Lidars, J. Atmos. Ocean. Tech., 23, 683-699, https://doi.org/10.1175/JTECH1872.1, 2006.

Bendix, J.: A Satellite-Based Climatology of Fog and Low-Level Stratus in Germany and Adjacent Areas, Atmos. Res., 64, 3-18, https://doi.org/10.1016/S0169-8095(02)00075-3, 2002.

Bennartz, R., Shupe, M. D., Turner, D. D., Walden, V. P., Steffen, K., Cox, C. J., Kulie, M. S., Miller, N. B., and Pettersen, C.: July 2012 Greenland Melt Extent Enhanced by Low-Level Liquid Clouds, Nature, 496, 83-86, https://doi.org/10.1038/nature12002, 2013.
Biele, J., Beyerle, G., and Baumgarten, G.: Polarization Lidar: Correction of Instrumental Effects, Opt. Express, 7, 427-435, https://doi.org/10.1364/OE.7.000427, 2000.

Cadeddu, M. P., Liljegren, J. C., and Turner, D. D.: The Atmospheric radiation measurement (ARM) program network of microwave radiometers: instrumentation, data, and retrievals, Atmos. Meas. Tech., 6, 2359-2372, https://doi.org/10.5194/amt-62359-2013, 2013.

Campbell, J. R., Hlavka, D. L., Welton, E. J., Flynn, C. J., Turner, D. D., Spinhirne, J. D., III, V. S. S., and Hwang, I. H.: FullTime, Eye-Safe Cloud and Aerosol Lidar Observation at Atmospheric Radiation Measurement Program Sites: Instruments and Data Processing, J. Atmos. Ocean. Tech., 19, 431-442, 2002.

Cesana, G. and Chepfer, H.: Evaluation of the Cloud Thermodynamic Phase in a Climate Model Using CALIPSOGOCCP, J. Geophys. Res.-Atmos., 118, 7922-7937, https://doi.org/10.1002/jgrd.50376, 2013.

Cesana, G., Kay, J. E., Chepfer, H., English, J. M., and de Boer, G.: Ubiquitous Low-Level Liquid-Containing Arctic Clouds: New Observations and Climate Model Constraints From CALIPSO-GOCCP, Geophys. Res. Lett., 39, L20804, https://doi.org/10.1029/2012GL053385, 2012.

Clothiaux, E. E., Moran, K. P., Martner, B. E., Ackerman, T. P., Mace, G. G., Uttal, T., Mather, J. H., Widener, K. B., Miller, M. A., and Rodriguez, D. J.: The Atmospheric Radiation Measurement Program Cloud Radars: Operational Modes, J. Atmos. Ocean. Tech., 16, 819-827, https://doi.org/10.1175/15200426(1999)016<0819:TARMPC>2.0.CO;2, 1999.

Curry, J. A., Schramm, J. L., Rossow, W. B., and Randall, D.: Overview of Arctic Cloud and Radiation Characteristics, J. Climate, 9, 1731-1764, https://doi.org/10.1175/15200442(1996)009<1731:OOACAR>2.0.CO;2, 1996.

de Boer, G., Eloranta, E. W., and Shupe, M. D.: Arctic Mixed-Phase Stratiform Cloud Properties from Multiple Years of SurfaceBased Measurements at Two High-Latitude Locations, J. Atmos. Sci., 66, 2874-2887, https://doi.org/10.1175/2009JAS3029.1, 2009.

Di, H., Hua, H., Cui, Y., Hua, D., Li, B., and Song, Y.: Correction Technology of a Polarization Lidar With a Complex Optical System, J. Opt. Soc. Am. A, 33, 1488-1494, https://doi.org/10.1364/JOSAA.33.001488, 2016.

Donovan, D. P., Whiteway, J. A., and Carswell, A. I.: Correction for Nonlinear Photon-Counting Effects in Lidar Systems, Appl. Optics, 32, 6742-53, 1993.

Flynn, C. J., Mendoza, A., Zheng, Y., and Mathur, S.: Novel Polarization-Sensitive Micropulse Lidar Measurement Technique, Opt. Express, 15, 2785-2790, https://doi.org/10.1364/OE.15.002785, 2007.

Freudenthaler, V.: About the effects of polarising optics on lidar signals and the $\Delta 90$ calibration, Atmos. Meas. Tech., 9, 41814255, https://doi.org/10.5194/amt-9-4181-2016, 2016.

Freudenthaler, V., Esselborn, M., Wiegner, M., Heese, B., Tesche, M., Ansmann, A., Mueller, D., Althausen, D., Wirth, M., Fix, A., Ehret, G., Knippertz, P., Toledano, C., Gasteiger, J., Garhammer, M., and Seefeldner, M.: Depolarization Ratio Profiling at Several Wavelengths in Pure Saharan Dust During SAMUM 2006, Tellus B, 61, 165-179, https://doi.org/10.1111/j.16000889.2008.00396.x, 2009. 
Fujii, T. and Fukuchi, T. (Eds.): Laser Remote Sensing, Taylor and Francis Group, Boca Raton, FL, USA, 2005.

Gimmestad, G. G.: Reexamination of Depolarization in Lidar Measurements, Appl. Optics, 47, 3795-3802, https://doi.org/10.1364/AO.47.003795, 2008.

Gregory, J. M., Huybrechts, P., and Raper, S. C. B.: Climatology: Threatened Loss of the Greenland Ice-Sheet, Nature, 428, 616616, https://doi.org/10.1038/428616a, 2004.

Groß, S., Freudenthaler, V., Wirth, M., and Weinzierl, B.: Towards an Aerosol Classification Scheme for Future EarthCARE Lidar Observations and Implications for Research Needs, Atmos. Sci. Lett., 16, 77-82, https://doi.org/10.1002/as12.524, 2015.

Hansen, J., Sato, M., Kharecha, P., and von Schuckmann, K.: Earth's energy imbalance and implications, Atmos. Chem. Phys., 11, 13421-13449, https://doi.org/10.5194/acp-11-13421-2011, 2011.

Hayman, M. and Thayer, J. P.: Explicit Description of Polarization Coupling in Lidar Applications, Opt. Lett., 34, 611-613, 2009.

Hayman, M. and Thayer, J. P.: General Description of Polarization in Lidar Using Stokes Vectors and Polar Decomposition of Mueller Matrices, J. Opt. Soc. Am. A, 29, 400-9, 2012.

Hoffmann, A., Ritter, C., Stock, M., Shiobara, M., Lampert, A., Maturilli, M., Orgis, T., Neuber, R., and Herber, A.: Ground-based lidar measurements from Ny-Ålesund during ASTAR 2007, Atmos. Chem. Phys., 9, 9059-9081, https://doi.org/10.5194/acp-99059-2009, 2009.

Intrieri, J. M., Shupe, M. D., Uttal, T., and McCarty, B. J.: An Annual Cycle of Arctic Cloud Characteristics Observed by Radar and Lidar at SHEBA, J. Geophys. Res.-Oceans, 107, SHE 5-1SHE 5-15, https://doi.org/10.1029/2000JC000423, 2002.

Kaul, B. V., Samokhvalov, I. V., and Volkov, S. N.: Investigating Particle Orientation in Cirrus Clouds by Measuring Backscattering Phase Matrices with Lidar, Appl. Optics, 43, 6620-6628, https://doi.org/10.1364/AO.43.006620, 2004.

Kay, J. E., Bourdages, L., Miller, N. B., Morrison, A., Yettella, V., Chepfer, H., and Eaton, B.: Evaluating and Improving Cloud Phase in the Community Atmosphere Model Version 5 Using Spaceborne Lidar Observations, J. Geophys. Res.-Atmos., 121, 4162-4176, https://doi.org/10.1002/2015JD024699, 2016.

Kjeldsen, K. K., Korsgaard, N. J., Bjørk, A. A., Khan, S. A., Box, J. E., Funder, S., Larsen, N. K., Bamber, J. L., Colgan, W., van den Broeke, M., Siggaard-Andersen, M.-L., Nuth, C., Schomacker, A., Andresen, C. S., Willerslev, E., and Kjær, K. H.: Spatial and Temporal Distribution of Mass Loss from the Greenland Ice Sheet Since AD 1900, Nature, 528, 396-400, 2015.

Klett, J. D.: Stable Analytical Inversion Solution for Processing Lidar Returns, Appl. Optics, 20, 211-220, https://doi.org/10.1364/AO.20.000211, 1981.

Liu, Z., Li, Z., Liu, B., and Li, R.: Analysis of Saturation Signal Correction of the Troposphere Lidar, Chin. Opt. Lett., 7, 10511054, 2009.

Measures, R. M.: Laser Remote Sensing: Fundamentals and Applications, John Wiley and Sons, Boca Raton, FL, USA, 1984.

Miller, N. B., Shupe, M. D., Cox, C. J., Walden, V. P., Turner, D. D., and Steffen, K.: Cloud Radiative Forcing at Summit Greenland, J. Climate, 28, 6267-6280, 2015.

Miller, N. B., Shupe, M. D., Cox, C. J., Noone, D., Persson, P. O. G., and Steffen, K.: Surface energy budget responses to radia- tive forcing at Summit, Greenland, The Cryosphere, 11, 497516, https://doi.org/10.5194/tc-11-497-2017, 2017.

Mishchenko, M. I. and Hovenier, J. W.: Depolarization of Light Backscattered by Randomly Oriented Nonspherical Particles, Opt. Lett., 20, 1356-1358, https://doi.org/10.1364/OL.20.001356, 1995.

Moran, K. P., Martner, B. E., Post, M. J., Kropfli, R. A., Welsh, D. C., and Widener, K. B.: An Unattended CloudProfiling Radar for Use in Climate Research, B. Am. Meteorol. Soc., 79, 443-455, https://doi.org/10.1175/15200477(1998)079<0443:AUCPRF>2.0.CO;2, 1998.

Morrison, H., de Boer, G., Feingold, G., Harrington, J., Shupe, M. D., and Sulia, K.: Resilience of Persistent Arctic Mixed-Phase Clouds, Nat. Geosci., 5, 11-17, https://doi.org/10.1038/ngeo1332, 2012.

Neely, R. R., Hayman, M., Stillwell, R. A., Thayer, J. P., Hardesty, R. M., O’Neill, M., Shupe, M. D., and Alvarez, C.: Polarization Lidar at Summit, Greenland for the Detection of Cloud Phase and Particle Orientation, J. Atmos. Ocean. Tech., 30, 1635-1655, https://doi.org/10.1175/JTECH-D-12-00101.1, 2013.

Newsom, R. K., Turner, D. D., Mielke, B., Clayton, M., Ferrare, R., and Sivaraman, C.: Simultaneous Analog and Photon Counting Detection for Raman Lidar, Appl. Opt., 48, 3903-3914, https://doi.org/10.1364/AO.48.003903, 2009.

Nott, G. J. and Duck, T. J.: Lidar Studies of the Polar Troposphere, Meteorol. Appl., 18, 383-405, https://doi.org/10.1002/met.289, 2011.

Pettersen, C., Bennartz, R., Kulie, M. S., Merrelli, A. J., Shupe, M. D., and Turner, D. D.: Microwave signatures of ice hydrometeors from ground-based observations above Summit, Greenland, Atmos. Chem. Phys., 16, 4743-4756, https://doi.org/10.5194/acp16-4743-2016, 2016.

Pithan, F., Medeiros, B., and Mauritsen, T.: Mixed-Phase Clouds Cause Climate Model Biases in Arctic Wintertime Temperature Inversions, Clim. Dynam., 43, 289-303, https://doi.org/10.1007/s00382-013-1964-9, 2014.

Sassen, K.: The Polarization Lidar Technique for Cloud Research: A Review and Current Assessment, B. Am. Meteorol. Soc., 72, 1848-1866, 1991.

Schotland, R. M., Sassen, K., and Stone, R.: Observations by Lidar of Linear Depolarization Ratios for Hydrometeors, J. Appl. Meteorol., 10, 1011-1017, https://doi.org/10.1175/15200450(1971)010<1011:OBLOLD>2.0.CO;2, 1971.

Sherwood, S. C., Bony, S., and Dufresne, J.-L.: Spread in Model Climate Sensitivity Traced to Atmospheric Convective Mixing, Nature, 505, 37-42, 2014.

Shupe, M. D.: Clouds at Arctic Atmospheric Observatories. Part II: Thermodynamic Phase Characteristics, J. Appl. Meteorol. Clim., 50, 645-661, https://doi.org/10.1175/2010JAMC2468.1, 2011.

Shupe, M. D. and Intrieri, J. M.: Cloud Radiative Forcing of the Arctic Surface: The Influence of Cloud Properties, Surface Albedo, and Solar Zenith Angle, J. Climate, 17, 616-628, https://doi.org/10.1175/15200442(2004)017<0616:CRFOTA>2.0.CO;2, 2004.

Shupe, M. D., Matrosov, S. Y., and Uttal, T.: Arctic Mixed-Phase Cloud Properties Derived from SurfaceBased Sensors at SHEBA, J. Atmos. Sci., 63, 697-711, https://doi.org/10.1175/JAS3659.1, 2006. 
Shupe, M. D., Daniel, J. S., de Boer, G., Eloranta, E. W., Kollias, P., Luke, E. P., Long, C. N., Turner, D. D., and Verlinde, J.: A Focus On Mixed-Phase Clouds, B. Am. Meteorol. Soc., 89, 1549-1562, 2008.

Shupe, M. D., Turner, D. D., Walden, V. P., Bennartz, R., Cadeddu, M. P., Castellani, B. B., Cox, C. J., Hudak, D. R., Kulie, M. S., Miller, N. B., Neely, R. R., Neff, W. D., and Rowe, P. M.: High and Dry: New Observations of Tropospheric and Cloud Properties above the Greenland Ice Sheet, B. Am. Meteorol. Soc., 94, 169-186, https://doi.org/10.1175/BAMS-D-11-00249.1, 2013.

Stevens, B. and Bony, S.: Water in the Atmosphere, Phys. Today, 66, 29-34, 2013.

Tan, I., Storelvmo, T., and Zelinka, M. D.: Observational Constraints on Mixed-Phase Clouds Imply Higher Climate Sensitivity, Science, 352, 224-227, 2016.

Thomas, L., Cartwright, J. C., and Wareing, D. P.: Lidar Observations of the Horizontal Orientation of Ice Crystals in Cirrus Clouds, Tellus B, 42, 211-216, https://doi.org/10.1034/j.16000889.1990.00001.x-i1, 1990.

Turner, D. D.: Arctic Mixed-Phase Cloud Properties from AERI Lidar Observations: Algorithm and Results from SHEBA, J. Appl. Meteorol., 44, 427-444, https://doi.org/10.1175/JAM2208.1, 2005.

Van De Hulst, H.: Light Scattering By Small Particles, John Wiley and Sons, New York, USA, 1957.

Van Tricht, K., Lhermitte, S., Lenaerts, J. T. M., Gorodetskaya, I. V., L/'Ecuyer, T. S., Noel, B., van den Broeke, M. R., Turner, D. D., and van Lipzig, N. P. M.: Clouds Enhance Greenland Ice Sheet Meltwater Runoff, Nat. Commun., 7, 10266, https://doi.org/10.1038/ncomms 10266, 2016.

Verlinde, J., Harrington, J. Y., Yannuzzi, V. T., Avramov, A., Greenberg, S., Richardson, S. J., Bahrmann, C. P., McFarquhar, G. M., Zhang, G., Johnson, N., Poellot, M. R., Mather, J. H., Turner, D. D., Eloranta, E. W., Tobin, D. C., Holz, R., Zak, B. D., Ivey, M. D., Prenni, A. J., DeMott, P. J., Daniel, J. S., Kok, G. L., Sassen, K., Spangenberg, D., Minnis, P., Tooman, T. P., Shupe, M., Heymsfield, A. J., and Schofield, R.: The Mixed-Phase Arctic Cloud Experiment, B. Am. Meteorol. Soc., 88, 205-221, https://doi.org/10.1175/BAMS-88-2-205, 2007.
Verlinde, J., Zak, B. D., Shupe, M. D., Ivey, M. D., and Stamnes, K.: The ARM North Slope of Alaska (NSA) Sites, Meteor. Mon., 57, 8.1-8.13, https://doi.org/10.1175/AMSMONOGRAPHS-D15-0023.1, 2016.

Walden, V. P., Shupe, M., Turner, D., and Bennartz, R.: Integrated Characterization of Energy, Clouds, Atmospheric State, and Precipitation at Summit (ICECAPS), Arctic Data Center, available at: https://arcticdata.io/catalog/\#view/urn:uuid: f783f072-e5e0-454c-9c9d-136935e1cb7c (last access: 1 August 2017), 2010.

Weitkamp, C. (Ed.): Lidar Range-Resolved Optical Remote Sensing of the Atmosphere, vol. 102, Springer, New York, NY, USA 2005.

Westbrook, C. D., Illingworth, A. J., O’Connor, E. J., and Hogan, R. J.: Doppler Lidar Measurements of Oriented Planar Ice Crystals Falling from Supercooled and Glaciated Layer Clouds, Q. J. Roy. Meteor. Soc., 136, 260-276, https://doi.org/10.1002/qj.528, 2010.

Whiteman, D. N.: Examination of the Traditional Raman Lidar Technique. I. Evaluating the TemperatureDependent Lidar Equations, Appl. Optics, 42, 2571-2592, https://doi.org/10.1364/AO.42.002571, 2003.

Whiteman, D. N., Melfi, S. H., and Ferrare, R. A.: Raman Lidar System for the Measurement of Water Vapor and Aerosols in the Earth's Atmosphere, Appl. Optics, 31, 3068-3082, https://doi.org/10.1364/AO.31.003068, 1992.

Winker, D. M., Vaughan, M. A., Omar, A., Hu, Y., Powell, K. A., Liu, Z., Hunt, W. H., and Young, S. A.: Overview of the CALIPSO Mission and CALIOP Data Processing Algorithms, J. Atmos. Ocean. Tech., 26, 2310-2323, 2009.

Zuidema, P., Baker, B., Han, Y., Intrieri, J., Key, J., Lawson, P., Matrosov, S., Shupe, M., Stone, R., and Uttal, T.: An Arctic Springtime Mixed-Phase Cloudy Boundary Layer Observed during SHEBA, J. Atmos. Sci., 62, 160-176, https://doi.org/10.1175/JAS-3368.1, 2005. 